\title{
Quand les choix comptables liés à l'environnement ne sont pas qu'opportunistes : cas des provisions comptables environnementales
}

\author{
Jonathan Maurice* \\ TSM-Research, Université Toulouse Capitole, CNRS
}

Pour citer cet article :

MAURICE (Jonathan), «Quand les choix comptables liés à l'environnement ne sont pas qu'opportunistes : cas des provisions comptables environnementales », Finance Contrôle Stratégie, mars 2019, vol. 22, $\mathrm{n}^{\circ} 1 ;<\underline{\text { https://journals.openedition.org/fcs/3197> }}$.

\section{Résumé}

Cet article étudie sous l'angle institutionnel la question des marges de manœuvre dont disposent les dirigeants et comptables pour estimer leurs provisions environnementales. Ces décisions ne pouvant être séparées du contexte social et technique dans lequel elles sont prises, une analyse qualitative du processus de détermination des provisions environnementales de groupes cotés français est menée pour mettre en évidence les éléments limitant ou favorisant l'utilisation opportuniste de ces montants. L'analyse des entretiens met en évidence l'importance des pressions institutionnelles (induisant un isomorphisme coercitif et mimétique dans le choix des méthodes d'estimation des provisions environnementales) qui rendent plus difficile l'utilisation discrétionnaire des provisions environnementales par les dirigeants. Ces résultats concluent sur l'intérêt de tenir compte, dans l'analyse, du contexte spécifique des décisions comptables en plus des incitations pouvant les engendrer.

Mots-clés : provisions environnementales, théorie néo-institutionnelle, isomorphisme, théorie positive de la comptabilité, opportunisme managérial, étude de cas.

\section{When environmental accounting choices are not only opportunistic: the case of environmental accounting provisions}

\author{
Abstract \\ From an institutional perspective, this paper examines the room for maneuver in estimating environmental \\ provisions. As the decisions of executives and accountants cannot be separated from the social and technical \\ context in which they are made, we conducted a qualitative study of French listed companies to investigate the
}


processes that are involved. Our objective was to determine which factors limit or encourage the opportunistic use of these provisional sums. The analysis of the interview transcripts revealed the impact of institutional pressure (leading to coercive and mimetic isomorphism in choosing the estimation method), which makes discretionary use of environmental provisions more difficult. These results indicate that an analysis has to take into account the specific context of the accounting decision, in addition to the incentives that give rise to it.

Keywords: environmental provisions, neo-institutional theory, isomorphism, positive accounting theory, opportunism, case study.

* Remerciements : je tiens à remercier les deux réviseurs anonymes ainsi que le rédacteur-en-chef Gérald Naro pour leurs remarques et conseils lors du processus de révision. L'article a également très largement bénéficié des commentaires de Charles H. Cho, Yves Dupuy, Sophie Giordano-Spring, Christophe Godowski, Michel Magnan et, en 2014, des participants de la $5^{\mathrm{e}}$ conférence italienne du Centre for Social and Environmental Accounting Research à Padoue et du $26^{\mathrm{e}}$ congrès international sur la recherche en comptabilité environnementale et sociale du CSEAR à St Andrews. Je remercie enfin Camille Gaudy pour son double codage des entretiens, ainsi que tous les participants professionnels de l'étude qui ont eu la gentillesse et le temps de me recevoir. Plus largement, ce projet de recherche a bénéficié du support financier de l'ANR GEODD (ANR-10-BLAN-1807), de la FNEGE dans le cadre du programme CEFAG et du programme « nouveaux entrants » de l'IDEX de l'université fédérale de Toulouse Midi-Pyrénées.

\section{Introduction}

La recherche comptable utilise régulièrement les données publiées par les entreprises dans leurs états financiers pour en déduire un comportement et attribuer une volonté précise, souvent opportuniste, aux dirigeants, indépendamment des caractéristiques spécifiques des données comptables en question. Il en est ainsi des éléments calculés du résultat, les accruals, dont la part discrétionnaire considérée dans son ensemble est utilisée par les entreprises pour, par exemple, gérer le résultat pour atteindre des seuils ou encore le lisser. Par extension, certains accruals pris isolément ont fait l'objet d'une étude particulière en raison de leur importance relative dans les comptes ou de leur nouveauté. C'est le cas des provisions à caractère environnemental (provisions pour remise en état de sites ou pour risques environnementaux) dont l'importance des montants et la forte incertitude entourant l'estimation des coûts environnementaux futurs en font un candidat raisonnable à la présomption de manipulation pour gérer le résultat. 
Dans cette optique, Berthelot et al. (2003) montrent par exemple que les entreprises cotées canadiennes utilisent leurs provisions environnementales pour lisser leur résultat comptable et prévenir l'émergence de coûts politiques. Peek (2004) conclut également à l'utilisation des provisions environnementales, et plus généralement des provisions, pour lisser le résultat comptable de l'entreprise. Johnston et Rock (2005) et Patten et Trompeter (2003) révèlent que, sous la menace d'une réglementation environnementale contraignante ou à la suite d'une catastrophe environnementale majeure, les entreprises ont tendance à diminuer leur résultat comptable de façon discrétionnaire (en utilisant notamment les provisions). Enfin, Schneider et al. (2017) mettent en évidence que les entreprises canadiennes ayant de fortes provisions environnementales utilisent stratégiquement un taux d'escompte supérieur afin de limiter l'augmentation de ces passifs au bilan.

Validant l'approche positive de la recherche en comptabilité, ces études quantitatives ne permettent pas de prendre en compte la complexité du processus organisationnel conduisant à l'estimation et la comptabilisation de telles provisions. Une première étude de Neu et Simmons (1996) sur les provisions pour remise en état de site rappelle l'importance de contextualiser les choix comptables, induits davantage par les relations sociales des managers et des comptables que par la recherche de leur intérêt personnel (à travers les incitations classiquement étudiées par la recherche positive en comptabilité). Dans cette perspective, cet article vise à étudier plus précisément le processus de détermination des provisions environnementales pour mettre en évidence les freins et facilitateurs institutionnels du comportement opportuniste des dirigeants dans l'estimation des provisions à caractère environnemental.

La question de recherche à laquelle cet article répond est donc la suivante : quels sont les éléments encourageant ou limitant l'usage discrétionnaire que peuvent faire les dirigeants des provisions environnementales ?

Pour y répondre de façon approfondie, une étude de cas multiple a été menée en 2011 sur la base d'entretiens semi-directifs avec les acteurs de dix groupes cotés français comptabilisant des provisions environnementales importantes, permettant ainsi d'étudier les organisations les plus concernées par cette problématique. Des commissaires aux comptes certifiant les comptes d'entreprises ayant de fortes provisions environnementales ont également été interrogés en tant qu'acteurs externes, mais informés, du processus de détermination des montants comptabilisés par les entreprises. Au total, 22 personnes ont été interrogées au sein de 13 organisations. Sur cette base, l'analyse met en évidence la technicité de l'estimation des coûts environnementaux associés aux provisions environnementales et le rôle important des services techniques des groupes dans ces estimations. En conséquence, les directions comptables doivent s'adapter à celles-ci et non les générer 
faute de compétences techniques, ce qui limite leur marge de manœuvre dans la comptabilisation des provisions. L'analyse des entretiens soutient également une lecture institutionnelle des comportements menant à la comptabilisation des provisions environnementales, notamment par la présence d'isomorphismes mimétique et coercitif. La lecture issue la théorie positive comptable dans laquelle l'opportunisme (de la gestion du résultat) guiderait la comptabilisation de ces provisions est peu soutenue par l'analyse, bien que l'approche qualitative ne soit pas la plus à même de faire émerger de tels comportements.

Cet article contribue ainsi à élargir l'explication des choix comptables influençant le résultat aux pressions institutionnelles qui s'exercent sur les acteurs et aux contraintes techniques auxquelles ils peuvent faire face. Il remet ainsi en question la seule explication fondée sur leur rationalité économique et la recherche de leur intérêt personnel, au profit d'une prise en compte plus large des conditions techniques et sociales dans lesquelles les choix comptables sont effectués et des contraintes institutionnelles qui pèsent sur les acteurs, en particulier les comptables et les dirigeants. À l'instar de Neu et Simmons (1996), l'article apporte un éclairage en profondeur d'un processus de décision comptable qui dépasse le cadre et les compétences seules des comptables et dirigeants, et dont l'issue ne peut donc être réduite à l'expression de leur opportunisme et rationalité.

Le plan de l'article se présente comme suit. La première section met la littérature étudiant les provisions environnementales en perspective des théories positive comptable et néo-institutionnelle. Elle présente également la méthode de recherche adoptée pour répondre à la question envisagée dans cette étude. La seconde section met en évidence les résultats de l'étude de cas multiple qui sont ensuite discutés. La conclusion résume enfin les résultats, apports et limites de l'étude.

\section{Provisions environnementales : demandes législatives, état de l'art, théories et méthodologie}

Cette première section présente d'abord le contexte comptable et fiscal de la comptabilisation des provisions environnementales par les groupes cotés français, sujets de l'étude. Elle synthétise ensuite la littérature focalisée sur les provisions environnementales majoritairement ancrée dans la théorie positive de la comptabilité. Une présentation des hypothèses et une discussion des limites de cette approche positive pour expliquer les choix relatifs aux provisions environnementales est ainsi initiée. De là, l'intérêt d'envisager ces choix sous l'angle néo-institutionnel est avancé en relation avec les avantages d'une approche qualitative de l'investigation empirique qui est alors présentée. 


\subsection{Demandes législatives}

Les provisions environnementales relèvent de la prise en compte des dépenses environnementales entendues comme «les dépenses effectuées en vue de prévenir, réduire ou réparer les dommages que l'entreprise a occasionnés ou pourrait occasionner par ses activités, à l'environnement ${ }^{1}$. Comptablement, ces dépenses environnementales peuvent être traitées de trois façons différentes :

1. Être comptabilisées directement en charges sans avoir été provisionnées préalablement.

2. Représenter un passif environnemental et donner lieu à dotation aux provisions pour risques et charges environnementales.

3. Être activées comme les coûts de démantèlement, d'enlèvement et de remise en état du site.

Les deux derniers cas se traduisent dans les comptes des entreprises par une provision environnementale. En normes françaises comme en normes internationales, cette distinction entre les provisions comptabilisées en contrepartie d'un actif et celles comptabilisées directement en résultat existe, et structure les définitions. Les provisions environnementales peuvent ainsi être distinguées et définies en fonction de leur traitement comptable basé sur les dépenses considérées : les dépenses environnementales de démantèlement, enlèvement et remise en état des sites qui seront portées à l'actif en contrepartie d'un passif environnemental puis amorties, et les autres dépenses environnementales provisionnables qui seront passées directement en résultat en contrepartie d'un passif environnemental.

Les groupes interrogés dans cette recherche étant tous cotés, ils appliquent le référentiel comptable international pour leurs comptes consolidés. Essentiellement, deux normes internationales et un commentaire de l'IFRIC concernent les provisions environnementales : l'IAS 37, l'IAS 16 et l'IFRIC 1. La norme IAS 37 aborde la comptabilisation des provisions, des actifs éventuels et des passifs éventuels. Une provision y est définie comme un passif dont l'échéance ou le montant est incertain sachant qu'un passif est une obligation actuelle de l'entité résultant d'événements passés et dont l'extinction devrait se traduire pour l'entité par une sortie de ressources représentatives d'avantages économiques. Cette obligation actuelle peut être une obligation juridique (contrat, lois, règlements, jurisprudence, etc.) ou une obligation implicite (lorsque «l'entreprise a indiqué aux tiers, par ses pratiques passées, par sa politique affichée ou par une déclaration récente suffisamment explicite, qu'elle assumera certaines responsabilités $»^{2}$ créant ainsi une attente fondée chez ces tiers).

1. Recommandation n ${ }^{0} 2003-r 02$ du 21 octobre 2003 du Conseil national de la comptabilité.

2. Extraits de la version française de la norme IAS 37 publiée au Journal officiel de l'Union européenne du 29 novembre 2008, L 320, p. 241-251. 
La norme IAS 16 concerne également la comptabilisation des provisions environnementales dans la mesure où elle traite de la comptabilisation des immobilisations corporelles dont les coûts comprennent «l'estimation initiale des coûts relatifs au démantèlement et à l'enlèvement de l'immobilisation et à la remise en état du site sur lequel elle est située, obligation qu'une entité encourt soit du fait de l'acquisition de l'immobilisation corporelle, soit du fait de son utilisation pendant une durée spécifique à des fins autres que la production de stocks au cours de cette période $\|{ }^{3}$. Pour l'évaluation de ces coûts inclus dans le montant activé de l'immobilisation corporelle concernée, la norme IAS 16 renvoie explicitement à la norme IAS 37. L'interprétation IFRIC 1 traite enfin de la «variation des passifs existants relatifs au démantèlement et à la remise en état, et des passifs similaires ». Elle précise le mode de comptabilisation de l'évolution des passifs liés aux remises en état par rapport à la norme IAS 37 et la norme IAS 16.

Il convient enfin d'indiquer que le processus de comptabilisation des provisions environnementales est double. D'abord, une estimation fiable des coûts associés aux obligations environnementales de l'entreprise doit être effectuée sur la base de compétences techniques propres aux activités de l'entreprise. Ensuite, la direction comptable doit déterminer si ces coûts sont provisionnables et, si oui, sous quelle forme doivent-ils être comptabilisés (avec la création d'un actif environnemental ou non). Enfin, une actualisation de la provision doit être également envisagée si l'horizon temporel est suffisamment long pour avoir un impact significatif sur celle-ci. Ces éléments de normalisation des provisions environnementales indiquent donc que celles-ci peuvent avoir un impact important sur le résultat comptable des entreprises, en dépit de leur caractère fortement estimatif. Les sous-sections suivantes présentent alors les cadres théoriques envisagés pour comprendre les décisions menant à leur comptabilisation.

\subsection{Provisions environnementales et théorie positive de la comptabilité}

Les provisions environnementales, éléments calculés des comptes pouvant influencer de manière significative le résultat des entreprises, ont majoritairement été étudiées par le prisme de la gestion du résultat. L'étude des comportements de gestion du résultat (earnings management) provient directement des objectifs de la théorie positive de la comptabilité de Watts et Zimmerman (1978, 1979, 1986) : «prédire et expliquer les choix comptables » des dirigeants (Watts et Zimmerman, 1990, p. 133). Pour ce faire, cette théorie emprunte essentiellement à la théorie de l'agence de Jensen

3. Extraits de la version française de la norme IAS 16 publiée au Journal officiel de l'Union européenne du 29 novembre 2008, L 320, p. 72-82. 
et Meckling (1976), aux modélisations économiques de la firme (Watts et Zimmerman, 1990) et à l'analyse micro-économique néoclassique (Boland et Gordon, 1992 ; Mouck, 1990). Elle rompt avec les approches théoriques normatives précédentes et a engendré un nombre important de recherches au point de devenir l'approche majoritaire dans de nombreuses revues comptables dont les plus prestigieuses aux États-Unis.

La théorie positive de Watts et Zimmerman (1986) repose sur plusieurs postulats issus des théories économiques et financières néoclassiques (Mouck, 1990), parmi lesquels : (a) les agents recherchent leur intérêt personnel (maximisation de leur utilité) et sont donc opportunistes dans leurs choix ; (b) l'entreprise est un nœud de contrats dans lequel les asymétries d'information renforcent la possibilité de comportements opportunistes ; (c) les marchés sont efficients et l'approche du MEDAF est valide. De ces postulats et emprunts de la théorie positive aux théories de l'agence (Jensen et Meckling, 1976) et des choix politiques (Posner, 1974) découlent une série de trois hypothèses principales testables visant à expliquer le comportement des dirigeants d'entreprise en matière de choix comptables. La comptabilité servant d'interface informationnelle entre les agents du nœud de contrats, les trois hypothèses concernent: (1) les dispositifs d'incitation des dirigeants (rémunération), (2) les conflits d'agence des dirigeants vis-à-vis des actionnaires et créanciers (via le ratio taux d'endettement / capitaux propres) et (3) les coûts politiques issus de réglementations défavorables mises en place essentiellement pour les grandes entreprises (une grande taille étant assimilée à un risque de monopole). Les hypothèses 1 et 2 impliquent que les dirigeants d'entreprises ayant un système d'intéressement ou un taux d'endettement élevé vont choisir les politiques comptables qui leur permettent de reporter des résultats futurs sur la période présente afin de capturer davantage de richesse des actionnaires et des créanciers. Les dirigeants des grandes entreprises doivent cependant arbitrer entre cette capture de richesse immédiate et le risque que de forts profits soient assimilés à une rente de monopole par le pouvoir politique. Ce dernier pourrait alors envisager de mettre en place une réglementation plus contraignante venant diminuer les profits futurs de l'entreprise et, par voie de conséquence, la rémunération des dirigeants recherchant leur intérêt personnel. Ainsi, cette troisième hypothèse de la théorie positive suppose que plus la taille d'une entreprise est grande, plus les dirigeants auront tendance à choisir les politiques comptables permettant de différer du résultat de l'exercice en cours vers les périodes futures afin d'éviter des coûts politiques.

Par conséquent, la théorie positive cherche à montrer que les dirigeants utilisent l'espace discrétionnaire dont ils disposent dans les normes comptables, plus ou moins permissives, pour augmenter leur richesse personnelle au détriment de la richesse des autres parties contractantes de 
l'entreprise. Le résultat comptable étant l'indicateur de performance privilégié dans de nombreux contrats entre dirigeants et parties prenantes de l'entreprise, l'étude des choix comptables relatifs aux provisions environnementales - en tant qu'éléments du résultat soumis au jugement des dirigeants a donc principalement été abordée sous cet angle théorique. Étant donné le caractère estimatif des provisions environnementales, les dirigeants peuvent, selon cette approche, ajuster le résultat comptable à la hausse ou à la baisse par ces éléments calculés afin d'atteindre un objectif de résultat (lissage, atteinte d'un résultat positif, atteinte des prévisions des analystes ou des objectifs annoncés). D'autre part, dans la perspective de réduire les coûts politiques subis par l'entreprise, les dirigeants peuvent également avoir intérêt à reporter dans le futur une partie des profits pour minimiser ces coûts de contractualisation politiques, et donc à comptabiliser des provisions environnementales plus importantes que nécessaire les premières années. C'est dans cette perspective théorique qu'une grande partie des précédentes études sur les provisions environnementales ont été menées. La décision de comptabiliser des provisions environnementales et le choix du montant inscrit dans les comptes sont supposés découler directement des arbitrages des dirigeants entre l'atteinte de certains seuils de résultat et la minimisation des coûts politiques environnementaux.

Dans cette optique, une première étude de Berthelot et al. (2003), spécifique aux provisions environnementales, analyse la relation entre les dotations aux provisions pour remise en état de sites et des variables usuellement utilisées pour tester les hypothèses issues de la théorie positive. Les auteurs se concentrent donc sur trois hypothèses dont deux sont directement issues de la théorie positive comptable pour expliquer les décisions des dirigeants en matière de provisions pour remise en état de sites. La première hypothèse testée avance que les dirigeants utilisent les provisions environnementales pour lisser le résultat comptable. Il est postulé que les dirigeants peuvent chercher à minimiser la variation du résultat comptable afin d'en présenter une évolution stable et positive ayant montré qu'elle pouvait augmenter la valeur de l'entreprise (Lev et Kunitzky, 1974) et diminuer le risque perçu (Farrelly et al., 1985). Une évolution régulière du résultat peut réduire le risque perçu par les investisseurs et donc augmenter artificiellement la valeur de l'entreprise. La deuxième hypothèse concerne le niveau d'endettement des entreprises supposé avoir un impact négatif sur les dotations aux provisions pour remise en état de sites. Plus l'entreprise est endettée et moins les dirigeants devraient avoir intérêt à diminuer leur résultat comptable pour respecter les clauses des contrats d'emprunt basées sur les résultats. La dernière hypothèse envisagée par les auteurs est celle de la visibilité politique de l'entreprise, initialement liée à sa taille mais pouvant être approximée par 


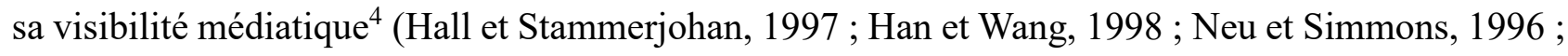
Watts et Zimmerman, 1986). Il est alors postulé que cette dernière peut inciter les dirigeants à diminuer leur résultat comptable par la dotation de provisions pour remise en état de sites, afin d'éviter d'éventuels coûts politiques futurs (renforcement de la réglementation environnementale, taxes environnementales, etc.). Sur un échantillon d'entreprises canadiennes cotées de 1990 à 1996 , les auteurs montrent que les dotations aux provisions pour remise en état de sites servent à lisser le résultat comptable et sont d'autant plus importantes que l'entreprise est visible médiatiquement. En revanche, l'hypothèse de l'endettement n'est pas vérifiée, conduisant d'une certaine manière à restreindre la validité de la théorie positive pour expliquer complètement les choix comptables relatifs aux provisions environnementales puisque les trois hypothèses formulées par Watts et Zimmerman (1978) sont théoriquement jointes (Milne, 2002). Parallèlement, cette étude montre, comme d'autres, que les provisions environnementales sont des informations utiles aux décisions des investisseurs (Baboukardos, 2017 ; Barth et McNichols, 1994 ; Berthelot et al., 2003 ; Bewley, 2005 ; Campbell et al., 2003 ; Clarkson et al., 2004 ; Li et McConomy, 1999 ; Wegener et Labelle, 2017), et méritent ainsi une considération particulière.

Quatre autres études incluant les provisions environnementales (entre autres) vont dans le sens des résultats de Berthelot et al. (2003). Tout d'abord, Patten et Trompeter (2003) étudient les choix comptables des entreprises chimiques américaines suite à l'explosion, fortement médiatisée, de l'usine d'Union Carbide à Bhopal en Inde en 1984. Ils montrent que, l'année de la catastrophe, les entreprises comptabilisent des accruals discrétionnaires négatifs et significatifs. Ces accruals discrétionnaires négatifs sont d'autant plus importants que la divulgation d'informations environnementales dans les rapports annuels était faible avant l'accident. Ensuite, Peek (2004) montre sur deux années que les provisions pour remise en état de sites, comme d'autres provisions, sont utilisées par les entreprises pour lisser leur résultat. Johnston et Rock (2005), s'ils n'étudient pas uniquement l'impact des dotations aux provisions environnementales sur le résultat, montrent également que les entreprises désignées par l'Agence de protection de l'environnement américaine comme parties potentiellement responsables de sites à dépolluer diminuent leurs résultats de manière discrétionnaire. Ces choix comptables s'expliqueraient alors par le fait que ces entreprises tentent d'éviter de payer la dépollution en raison du principe pollueur-payeur mis en place au départ par l'Agence, les entreprises étant considérées comme solidairement responsables de la pollution d'un

4. Il faut noter ici que le test de l'hypothèse des coûts politiques par la visibilité politique (approximée par la visibilité médiatique) de l'entreprise est critiquable et critiquée (Milne, 2002) dans la mesure où une entreprise très visible n'est pas forcément très profitable ni sujette à de forts coûts politiques tels qu'envisagés par Watts et Zimmerman (1978). 
site dès lors qu'elles y avaient contribué. Enfin, Schneider et al. (2017) illustrent dans le cas canadien l'utilisation discrétionnaire du taux d'escompte des provisions environnementales pour limiter l'augmentation de ces passifs au bilan. La détermination du taux d'escompte des provisions environnementales, laissé à la libre appréciation de l'entreprise dans le contexte réglementaire canadien, résulte donc d'un choix stratégique des dirigeants souhaitant maitriser l'image comptable de leur entreprise.

Si la théorie positive de la comptabilité fournit un des cadres d'analyse possibles des choix comptables relatifs aux provisions environnementales, elle n'est pas complètement satisfaisante à plusieurs égards. Tout d'abord, ses fondements micro-économiques postulant que les dirigeants ne font que des choix maximisant leur intérêt personnel sont critiquables et théoriquement incompatibles avec l'existence de marchés en déséquilibre (Boland et Gordon, 1992). Ensuite, d'autres facteurs comportementaux, mais aussi sociaux et organisationnels peuvent contraindre et expliquer les choix comptables relatifs aux provisions environnementales, en dehors de la maximisation des intérêts des dirigeants. Enfin, l'hypothèse des coûts politiques, en particulier, souffre de limites méthodologiques et théoriques (Milne, 2002). C'est dans la perspective de pallier ces limites que la théorie néoinstitutionnelle peut être mobilisée pour fournir un cadre complémentaire d'analyse du processus de décision conduisant aux provisions environnementales.

\subsection{Provisions environnementales et théorie néo-institutionnelle}

1.3.1. Approche néo-institutionnelle dans la littérature comptable

En préambule, un parallèle peut être fait entre l'hypothèse des coûts politiques et l'approche néoinstitutionnelle dans la mesure où les deux postulent que «les réactions des entreprises sont destinées à réduire ou éviter l'intervention politique, la régulation et les coûts en découlant» (Milne, 2002, p. 384, trad. de l'auteur). Ainsi, les pressions institutionnelles sont, dans les deux paradigmes, à l'origine de ces réactions d'entreprises. La différence principale réside dans le fait que l'hypothèse des coûts politiques associe les choix comptables à un comportement maximisateur des dirigeants cherchant à se prémunir d'une perte de revenus liées à ces pressions institutionnelles, tandis que l'approche néo-institutionnelle interprète les choix comptables comme le résultat de comportements collectifs, souvent ritualisés et ancrés dans le monde social des individus qui y concourent.

Sur la base de ces différences fondamentales, et dans une perspective critique de la théorie positive comptable, Neu et Simmons (1996) soutiennent que cette dernière est insuffisante pour expliquer les choix comptables des dirigeants qui ne sont pas de simples agents maximisateurs indépendants du contexte social dans lequel ils évoluent. En décontextualisant et réduisant les comportements des 
dirigeants à de simples calculs maximisateurs, la théorie positive comptable ne permettrait pas, à elle seule, d'en analyser et d'en comprendre toute la complexité. D'autres interprétations des choix comptables sont alors envisageables, comme par exemple celles de l'approche néo-institutionnelle qui suggère «que les managers agissent souvent d'une façon rituelle plutôt que d'une façon rationnelle et opportuniste » (p. 411 et 412, trad. de l'auteur). Pour illustrer l'importance d'élargir l'analyse aux relations sociales des dirigeants, Neu et Simmons (1996) étudient leurs choix comptables relatifs aux provisions pour remise en état de sites en mobilisant à la fois l'analyse de données comptables secondaires et d'entretiens conduits sur le terrain auprès des managers faisant face à ce type de provisions dans leur entreprise. L'utilisation d'entretiens qualitatifs leur permet d'enrichir l'explication des choix comptables effectués par les dirigeants et de valider les variables incluses dans l'analyse quantitative.

À travers les entretiens, les auteurs montrent ainsi, par exemple, l'importance des commissaires aux comptes dans le choix du montant comptabilisé au titre de la remise en état de sites. En ce qui concerne le choix d'une méthode comptable prospective ou rétrospective à la première application de la norme comptable impliquant la comptabilisation des coûts de remise en état de sites, les entretiens et l'analyse quantitative révèlent que les managers préfèrent choisir la méthode comptable qui minimise les contraintes potentielles des relations sociales dans lesquels ils sont intégrés (avec les investisseurs, les banquiers, les pouvoirs politiques, les employés). L'analyse se veut plus riche par les entretiens menés, et réintroduit la question de la complexité des choix et des comportements des managers qui ne sont pas uniquement des agents maximisateurs extraits du contexte social. En interrogeant des managers au sujet du processus de décision menant à la comptabilisation des provisions environnementales, il est possible de mettre en évidence les facteurs sociaux et organisationnels influençant ces choix comptables, en dehors du seul intérêt personnel des dirigeants. La théorie néo-institutionnelle insiste davantage sur l'aspect rituel plutôt que rationnel des actions des dirigeants, dans la mesure où « les acteurs [ne sont] pas [toujours] en mesure de reconnaître ou d'agir selon leurs intérêts » (DiMaggio, 1988, p. 105, trad. de l'auteur). Mezias (1990) développe par exemple un modèle empirique permettant de tester à la fois les hypothèses issues des arguments économiques de maximisation des agents et les hypothèses institutionnelles sur le choix de pratiques de reporting financier. Il montre que, si les hypothèses économiques expliquent une partie des choix comptables, les hypothèses institutionnelles (isomorphisme coercitif ou institutionnel, entre autres) en expliquent une plus grande partie, concluant que «les comportements organisationnels sont affectés par l'environnement institutionnel et pas seulement par les caractéristiques de l'entreprise » (p. 451, trad. de 1'auteur). 
Dans un autre contexte, Touron (2004) montre que l'adoption volontaire d'un jeu de normes comptables de remplacement peut s'expliquer à la fois par un problème d'agence et par des influences normatives et mimétiques issues du contexte institutionnel de l'entreprise. À travers une étude de cas de Saint-Gobain-Pont-à-Mousson, l'auteur met alors en évidence que le choix d'adoption des US GAAP par l'entreprise dans les années 1970 résulte davantage d'une volonté de se légitimer en tant qu'acteur international souhaitant pénétrer le marché américain que d'une tentative de réduire les conflits d'agence entre dirigeants, actionnaires et banquiers. Les influences normatives des auditeurs américains ayant contribué à la mise en place des US GAAP chez Saint-Gobain-Pont-à-Mousson, ainsi que les influences mimétiques des grandes entreprises américaines avec lesquelles voulaient se comparer les dirigeants, ont permis à l'auteur d'expliquer les motivations de l'entreprise à l'adoption de normes comptables de remplacement. La théorie néo-institutionnelle est ainsi considérée comme complémentaire de l'approche économique de la théorie de l'agence puisque tout choix comptable se prend dans un contexte institutionnel particulier. Dans la continuité, Barbu et Piot (2012) ont mené une étude quantitative auprès de directeurs financiers de grands groupes cotés français concernant l'adoption des normes IAS-IFRS. S'appuyant également sur la théorie néo-institutionnelle, ils montrent que le passage aux normes internationales a été perçu par les directeurs financiers comme essentiellement contraint, traduisant l'influence coercitive du règlement européen 1606/2002 soutenu par le normalisateur français. Ils révèlent par ailleurs que ce passage aux normes internationales a également été un moyen pour les entreprises de se légitimer, par mimétisme des entreprises jugées plus performantes. En revanche, ils mettent en évidence que cette adoption n'a pas été considérée comme permettant d'améliorer la performance économique (par diminution des coûts ou amélioration de l'efficacité de l'information financière), limitant ainsi la pertinence des explications issues du paradigme d'efficience économique de la théorie positive de la comptabilité. Il faut toutefois nuancer cette conclusion lorsqu'on la transpose à la gestion du résultat car les décisions associées à celle-ci n'impliquent pas forcément une amélioration de l'efficience économique à long terme (dans le cas d'une restriction des dépenses de recherche et développement pour gérer le résultat à la hausse à court terme, par exemple).

Sur la base de ces différentes études, il est intéressant d'envisager d'autres facteurs, d'ordre institutionnel, pouvant influencer la comptabilisation des provisions environnementales, comme les pratiques de la maison-mère, la promotion d'une méthode comptable par les associations industrielles lorsque l'incertitude est forte (DiMaggio et Powell, 1983), la pression des commissaires aux comptes (Neu et Simmons, 1996) ou de l'administration (chargée de l'environnement, fiscale...), les normes de l'industrie ou du secteur (Neu, 1992), les conseillers professionnels (comme les experts- 
comptables, les consultants, les avocats, etc.), les agences de notations ou les assureurs. Negash (2012) met par exemple en évidence une convergence des comportements de (non)-divulgation des provisions environnementales dans les rapports annuels de trois entreprises pétrolières : pas de divulgation claire des montants provisionnés et une information qualitative similaire (très succincte) d'une entreprise à l'autre. L'auteur évoque alors l'isomorphisme institutionnel normatif (DiMaggio et Powell, 1983) du métier de l'exploitation pétrolière, poussant les entreprises à adopter les mêmes pratiques de divulgation que leurs concurrentes pour maintenir leur légitimité. La comptabilisation de provisions environnementales et leur diffusion dans les comptes annuels peuvent ainsi servir d'outils de légitimation dans la mesure où celles-ci permettent d'indiquer aux parties prenantes que l'entreprise prend en compte, et assume, sa responsabilité en matière d'environnement.

\subsubsection{Analyse des choix comptables par les concepts d'isomorphisme et de légitimité}

La théorie néo-institutionnelle (DiMaggio et Powell, 1983 ; Meyer et Rowan, 1977) permet d'expliquer les changements organisationnels contribuant à l'adoption par les organisations de structures et comportements similaires. Elle permet de rendre compte de décisions prises collectivement par un champ organisationnel plutôt que par un agent économique isolé (Barbu, 2006). Sur ce champ organisationnel constitué des organisations formant un espace de vie institutionnelle reconnu s'exercent différentes pressions institutionnelles. Les organisations du champ vont alors y répondre de façon homogène pour maintenir la légitimité de leurs activités, prenant des décisions allant parfois à l'encontre de la rationalité économique. En particulier, trois pressions institutionnelles permettent d'expliquer ces tendances d'homogénéisation : l'isomorphisme coercitif, l'isomorphisme normatif et l'isomorphisme mimétique.

L’isomorphisme coercitif est défini par DiMaggio et Powell (1983, p. 150) comme le « résultat de pressions formelles et informelles exercées sur les organisations par d'autres organisations dont elles dépendent et par les attentes culturelles de la société dans laquelle ces organisations opèrent ». Les organisations ont donc tendance à adopter des structures et des comportements conformes aux exigences formulées par d'autres organisations ayant le pouvoir de contrôler et sanctionner les déviances à ce qui est légitimement attendu par ces dernières (ce qu'exprime la notion de dépendance). Par exemple, l'environnement légal, qui est commun à de nombreuses organisations, contribue à normaliser les comportements et les structures organisationnelles. L'État et les organisations le représentant exercent par ce biais une influence coercitive sur les entreprises. Touron (2004, p. 175) cite par exemple deux organisations susceptibles d'exercer des pressions coercitives au niveau comptable : l'État et les commissions de réglementation boursière. De même, les cabinets 
d'audit légal exercent une influence coercitive sur les organisations dans la mesure où ils possèdent un mécanisme de sanction formel sur l'entreprise, la non certification des comptes ou la certification avec réserve. Ils émettent un avis d'expert sur lequel d'autres organisations peuvent prendre appui pour juger la légitimité de l'entreprise (à travers sa représentation comptable). L'audit légal repose sur de nombreux comportements ritualisés et, comme l'indiquent Neu et Simmons (1996, p. 415), « en termes de légitimité, le fait de ne pas suivre les conseils de professionnels pourrait être interprété comme coercitif $\gg$. Le caractère fortement estimatif des provisions environnementales et leur importance souvent significative sont susceptibles d'inciter les commissaires aux comptes à rechercher (et valider seulement) des méthodes d'estimation et de comptabilisation connues et comparables d'une entreprise à l'autre. Les entreprises peuvent donc adopter des méthodes similaires sous la pression de leur cabinet d'audit légal.

L'isomorphisme normatif est associé par DiMaggio et Powell (1983) à la professionnalisation, contribuant à homogénéiser les comportements des membres d'une profession en fournissant le cadre des conditions et méthodes de travail considérées comme légitimes par celle-ci. Ils insistent notamment sur deux aspects de la professionnalisation qui favorisent l'isomorphisme normatif. Les comportements professionnels sont d'abord façonnés par ce qui reste de la base cognitive acquise et légitimée par les individus pendant leur éducation formelle à l'université. Ensuite, « la croissance et l'élaboration des réseaux professionnels qui transcendent les organisations et à travers lesquels les nouveaux modèles se diffusent rapidement » (p. 152) contribuent à l'homogénéité des comportements dans les organisations. Les règles de fonctionnement privilégiées et légitimées par la profession comptable et la profession des ingénieurs environnement devraient donc se retrouver d'une entreprise à l'autre. Une certaine homogénéité des méthodes et procédures d'estimation et de comptabilisation des provisions environnementales peut ainsi venir des normes diffusées par les cabinets de conseil en environnement, les cabinets d'audit durant leurs missions de conseil ou par les cercles de rencontre de la profession comptable dans lesquels l'information peut transiter.

L'isomorphisme mimétique peut quant à lui être à l'œuvre en présence d'incertitude, comme « lorsque l'organisation fait face à un problème dont les causes sont ambiguës ou les solutions peu claires » (DiMaggio et Powell, 1983, p. 151, trad. de l'auteur). Ainsi, l'imitation d'autres entreprises perçues comme légitimes ou plus performantes est un moyen pour l'organisation de réduire cette incertitude et ainsi de maintenir sa légitimité en adoptant des solutions, considérées comme efficaces, à moindre coût. Dans le cas des provisions environnementales, l'incertitude entourant l'estimation des coûts futurs de remise en état de site ou de dépollution peut conduire les entreprises à s'imiter au sein d'une industrie. L'accès à l'information sur les méthodes employées par les autres entreprises 
perçues comme légitimes ou plus performantes sur ces sujets-là peut passer par les cercles professionnels, les contacts individuels des comptables et ingénieurs dans d'autres entreprises, ou encore par le transfert d'employés très qualifiés (ingénieurs environnement par exemple) d'une entreprise à l'autre.

Ces différentes pressions conduisant à l'homogénéisation des structures et des comportements des organisations ont parfois des frontières floues, et sont souvent cumulatives. Le commissaire aux comptes peut exercer à la fois une pression coercitive et normative sur l'organisation en fonction de sa mission (audit légal ou conseil). L'isomorphisme mimétique est parfois favorisé parce que les individus partagent de nombreuses normes professionnelles et peuvent échanger de l'information audelà de leur organisation. Dans le cas des provisions environnementales, la mise en évidence des acteurs, internes comme externes, participant ou influençant leur processus d'estimation et de comptabilisation peut permettre de révéler les enjeux de cette comptabilisation et les pressions que peuvent subir les managers, pour ensuite expliquer leurs comportements et incitations. À travers des entretiens semi-directifs, il est possible de décrire et d'analyser le processus de décision menant aux provisions environnementales.

\subsection{Méthodologie}

Une étude de cas multiple a été conduite à travers des entretiens semi-directifs d'acteurs connaissant des provisions environnementales dans leur métier. Vingt-deux personnes appartenant à treize organisations ont été interrogées en 2011. Les sous-sections suivantes présentent la démarche de constitution et les caractéristiques de cet échantillon (section 1.4.1), ainsi que la démarche d'analyse des données (section 1.4.2).

\subsubsection{Démarche de contact et description de l'échantillon des personnes interrogées}

L'étude menée est une étude de cas multiple portant sur dix organisations comptabilisant des provisions environnementales, auxquelles il faut ajouter les entretiens avec des commissaires aux comptes de trois cabinets d'audit. Yin (2009, p. 58) indique que si le chercheur souhaite un fort degré de certitude sur ses résultats, au moins cinq ou six réplications littérales, voire plus, peuvent être nécessaires, ce qui est le cas ici. Par ailleurs, la saturation des données (traduisant le fait que les informations supplémentaires obtenues n'apportent plus d'informations nouvelles sur le phénomène étudié) a été atteinte sur les thèmes principaux suivants : étapes du processus de détermination des provisions environnementales, acteurs impliqués, enjeux des provisions environnementales, impact de la réglementation et recherche de légitimité. 
Déterminer les personnes à interroger s'est fait en deux étapes. La première étape a consisté en la sélection des organisations pertinentes à l'analyse d'un processus de détermination des provisions environnementales, la seconde étape en la sélection des personnes potentiellement liées au processus étudié au sein de ces organisations. À la première étape, les groupes cotés français du SBF 250 et des secteurs sensibles aux risques environnementaux ont été classés par ordre décroissant de leurs provisions environnementales au bilan de l'exercice 2010 et sélectionnés en fonction de l'importance des montants comptabilisés. L’annexe 1 présente les caractéristiques des groupes interrogés.

Les entretiens ont été menés de façon systématique à des fins de comparaison (guides d'entretien similaires mais adaptés à chaque catégorie de personnes interrogées). Les annexes $3 \mathrm{a}$ et $3 \mathrm{~b}$ présentent les guides d'entretien à destination des directeurs financiers et des commissaires aux comptes. Celui à destination des directeurs du développement durable est proche de celui à destination des directeurs financiers et n'est pas reporté. Les sections 2 à 4 (ou 5) des guides d'entretien présentent des questions permettant de comprendre l'influence de facteurs externes (acteurs ou réglementations) sur les décisions de provisionnement (approche néo-institutionnelle). Les réponses aux questions visant à préciser les étapes du processus de décision et celles concernant le processus d'audit ont permis de faire émerger certains indices de gestion ou non gestion du résultat (approche positive). L'accès au terrain s'est fait par contact direct ${ }^{5}$, par parrainage pour deux entretiens et par connaissance pour un entretien. Étant donné que les entretiens au sein d'une organisation ont été réalisés sur une courte période de temps en 2011, les phénomènes de contamination des personnes interrogées sont très faibles. En revanche, il existe probablement un biais lié au fait que seules les personnes volontaires ont pu être interrogées.

Les entretiens semi-directifs ont été menés avec vingt-deux personnes appartenant à treize organisations différentes dans les secteurs suivants : automobile, gestion de déchets et eau, pétrole, extraction de minerais, télécommunications, pharmaceutique, chimie et audit. Les annexes 1 et 2 précisent les postes occupés par les personnes interrogées au sein des groupes étudiés et la durée des entretiens. Sur ces vingt-deux personnes :

- quatre font partie du métier de l'audit (deux commissaires aux comptes de deux grands cabinets certifiant les comptes de groupes ayant de fortes provisions environnementales ; un expertcomptable et commissaire aux comptes et sa collaboratrice, impliqués au club développement durable de l'Ordre des experts-comptables);

5. Ce contact direct a parfois donné lieu à un entretien collectif avec des personnes non contactées dans un premier temps, assimilable à du parrainage. 
- six exercent une profession comptable au sein de la maison-mère ou d'une filiale d'un groupe coté français ayant des provisions environnementales (deux directeurs administratifs et financiers - d'un groupe et d'une filiale étrangère - ; un directeur des normes comptables avec l'un de ses collaborateurs ; un directeur de la comptabilité et un directeur de la consolidation et du reporting);

- huit ont un rôle dans la politique environnementale du groupe (trois directeurs du développement durable et l'assistant de l'un d'eux ; un directeur environnement ; un directeur de la réhabilitation environnementale qui s'occupe spécifiquement des provisions environnementales du groupe ${ }^{6}$; un directeur hygiène, santé et sécurité ; un directeur des indicateurs et de la performance environnementale, sociale et de gouvernance) ;

- deux sont spécialisées dans le reporting au niveau du groupe (l'une dans le reporting sécurité et accident et l'autre dans le reporting environnement, étant à l'origine de sa mise en place) ;

- une est directeur des risques ;

- une est directeur de l'innovation, de la recherche et la technologie et travaille en étroite collaboration avec la direction financière au sujet des provisions environnementales.

Plusieurs avantages découlent de l'échantillon des personnes interrogées. La plupart de ces personnes a une bonne connaissance des problématiques liées aux provisions environnementales. Celles indiquant ne pas l'avoir ont toutefois permis de préciser quels types d'acteurs se trouvaient complètement à l'extérieur du processus. Toutes les personnes interrogées, sauf une, appartiennent à la maison-mère de leur groupe, assurant un haut niveau de décision et une vue d'ensemble des provisions environnementales au sein du groupe. Les postes (et profils) des personnes interrogées sont également suffisamment variés pour préciser la place de chacun dans le processus et recouper les informations obtenues d'un poste à l'autre. Dans certains cas, plusieurs personnes (à des postes différents) du même groupe ont pu être interrogées conjointement ou indépendamment, assurant une description plus précise du processus de décision entourant les provisions environnementales de ces groupes. La présence de commissaires aux comptes spécialisés dans les questions environnementales et certifiant les comptes de groupes ayant des provisions environnementales fortes permet d'assurer un regard extérieur, à la fois informé et relativement indépendant, sur le processus de détermination des provisions environnementales.

6. De la documentation interne sur les procédures de comptabilisation des provisions environnementales du groupe chimique (feuilles Excel de reporting semestriel, diapositives de présentation du processus de réhabilitation) est venue supporter le discours du directeur de la réhabilitation environnementale concernant leur processus de détermination des provisions. 


\subsubsection{Démarche d'analyse des données qualitatives collectées}

Les entretiens ont été retranscrits puis codés sur la base de catégories existant a priori, à l'aide du logiciel NVivo 9. L'analyse des entretiens est alors thématique et repose sur un codage similaire à celui présenté par Miles et Huberman (1984). Les catégories préexistantes à l'analyse sont issues majoritairement du guide d'entretien et des thèmes qui y sont abordés dans la mesure où il s'agissait d'abord de décrire le processus de détermination des provisions environnementales. Sont également ajoutées des catégories issues de la théorie positive de la comptabilité, avec notamment des catégories ayant trait à la gestion du résultat ou à la tromperie comptable, et des théories néo-institutionnelles, avec des catégories regroupant spécifiquement les assertions liées à la recherche de légitimité des groupes ou à leur isomorphisme. En fonction des thèmes annexes abordés avec les personnes interrogées, des catégories ont pu émerger a posteriori de manière marginale comme les relations entre l'investissement et la politique de développement durable du groupe ou des aspects de performance environnementale. Le codage des entretiens a été effectué deux fois par le chercheur, lors de la première analyse des données pour produire une version initiale des résultats puis, plusieurs mois après, préalablement à la rédaction de cet article. L'annexe 4 présente les nœuds retenus à l'issue du codage. De plus, un double codage partiel sur un échantillon d'entretiens représentatif des acteurs interrogés a été effectué par un autre chercheur spécialisé en comptabilité et audit environnemental. Le résultat de ce double codage est venu confirmer les premières analyses concernant les catégories préexistantes, ainsi que l'identification et le rôle des acteurs émergeant des entretiens.

Les catégories utilisées dans cette recherche permettent de détailler les éléments récurrents du processus de détermination des provisions environnementales, et de circonscrire les acteurs y participant ainsi que ceux n'y participant pas. Les réponses des personnes interrogées sont analysées dans une perspective de triangulation des informations obtenues sur le processus et les personnes y participant dans la mesure où plusieurs organisations et plusieurs personnes à différents stades du processus ont été interrogées.

\section{Provisions environnementales : pressions institutionnelles et choix comptables}

Les résultats de l'étude qualitative mettent en évidence plusieurs éléments cadrant les prises de décisions autour des provisions environnementales. Si ces résultats ne permettent pas de montrer comment le champ organisationnel (toutes les organisations et acteurs impliqués) s'institutionnalise 
sur la durée, ils permettent d'en donner une description à l'instant $t$. Il est alors possible de mettre en évidence les pressions institutionnelles qui sont susceptibles aujourd'hui de conduire les entreprises à adopter des comportements similaires dans l'estimation et la comptabilisation des provisions environnementales, et donc de contraindre les comportements opportunistes isolés. La description de ces influences institutionnelles contribue à expliquer pourquoi les comportements opportunistes des dirigeants sont limités dans l'utilisation des provisions environnementales à des fins personnelles, contrairement aux précédentes études basées sur la théorie positive. Ainsi, l'étude du processus de détermination des provisions environnementales et les acteurs y participant révèlent d'abord que les directions comptables et les dirigeants n'en seraient pas les acteurs déterminants (section 2.1). Ensuite, un certain nombre de pressions institutionnelles créent les conditions d'un isomorphisme coercitif et mimétique (section 2.2). Enfin, il apparaît que les pratiques de gestion du résultat comptable à travers les provisions environnementales restent possibles mais limitées. Les techniques d'évaluation des risques environnementaux contraignent assez fortement l'étendue des valeurs considérées et, par là-même, les manipulations possibles des montants comptabilisés (section 2.3). Une discussion de ces résultats est avancée dans une dernière section (2.4).

\subsection{Acteurs internes et externes influençant le processus de détermination des provisions environnementales}

Cette première section présente les acteurs, internes puis externes, qui influencent le processus de détermination des provisions environnementales, et qui contribuent à définir le champ organisationnel dans lequel s'inscrivent les entreprises. Elle synthétise le processus d'estimation et de comptabilisation des provisions environnementales tel qu'il a été décrit par les personnes interrogées.

\subsubsection{Acteurs internes participant à la détermination des provisions environnementales}

Au sein d'un groupe de sociétés, le processus de détermination des provisions environnementales part d'abord des services techniques spécialisés en environnement et en remise en état de sites (parmi lesquels des géologues, des ingénieurs des mines, des contrôleurs de gestion opérationnels, etc.) de la maison-mère et des filiales concernées (cabinets d'études internes pour les grands groupes, parfois externes) avec lesquels les commissaires aux comptes font la revue des provisions (voir ci-après). Ces services sont en général indépendants des donneurs d'ordre opérationnels.

Le deuxième acteur-clé du processus de détermination des provisions environnementales est la direction financière du groupe qui essaye de prendre les solutions «les moins douteuses » en termes 
de provisions environnementales et d'avoir une bonne connaissance des obligations réglementaires environnementales ${ }^{7} \mathrm{du}$ groupe. Les directeurs financiers locaux sont au départ du processus, conjointement avec les services techniques sur le terrain.

« Notre rôle, c'est d'avoir une bonne vision des risques et de bien être confortable sur le fait qu'on a des provisions adéquates et que ces risques sont bien identifiés. Leur rôle [aux managers des sites], c'est le même sur le terrain mais en plus eux ils sont confrontés à l'opérationnel quotidien. »

(Directeur de l'innovation d'un groupe d'extraction de minerais.)

Un dernier acteur-clé est apparu spécifiquement au sein du groupe chimique étudié, dont le processus est apparu le plus abouti des groupes de l'échantillon de personnes interrogées. Il s'agit du directeur de la réhabilitation environnementale qui est chargé spécifiquement de l'évaluation des sorties de ressources liées aux risques environnementaux et aux remises en état de sites. Le processus de détermination des provisions environnementales de ce groupe est centralisé sur cet acteur qui est in fine en lien avec la direction comptable pour la transcription en comptabilité de ces flux monétaires (c.-à-d. la transformation en provisions). Le directeur de la réhabilitation environnementale dispose d'une équipe dédiée distincte des opérationnels qui, eux, ne gèrent pas les provisions. Cette équipe comprend les correspondants en charge de la réhabilitation (sur chacun des sites encore en activité de même que sur les sites fermés) et les membres du service au sein de la maison-mère. Les relais de cette équipe et du directeur de la réhabilitation environnementale sont les responsables hygiène, sécurité et environnement des sites.

À côté de ces acteurs principaux, d'autres composantes de l'organisation interviennent de façon plus ponctuelle dans le processus étudié. C'est le cas des dirigeants et des responsables du contrôle de gestion qui interviennent dans un dialogue avec les directions comptables pour valider (être informé de, au minimum) les estimations reposant sur les données des services techniques opérationnels.

\footnotetext{
«Le directeur financier, ou le directeur général d'ailleurs, n'interviendront que si les techniciens, que ce soit les techniciens locaux ou les techniciens centraux [...] ou même les techniciens centraux entre eux, ou les techniciens locaux entre eux, ne sont pas d'accord. Souvent, il interviendra parce qu'il faut bien que quelqu'un prenne la décision. [...] Les techniciens vont donner un chiffre. Il sera un peu « challengé » bien entendu. Mais c'est celui qui sera pris. »

(Commissaire aux comptes.)
}

7. Les juristes interviennent à ce niveau du processus, en consultation seulement pour connaître l'état des obligations contractuelles et légales de remise en état de sites et de responsabilité environnementale. 
«Ces provisions environnement sont validées d'un point de vue technique et d'un point de vue financier. Tous les organes de décision ne peuvent pas être dans les mêmes mains. Donc justement, on s'attache à avoir un processus où moi je fais ma propre évaluation et où je propose mes cash-flows sur la base d'hypothèses ou d'évaluations que je fais et par contre après, tout ça, c'est validé avec la finance. Puisqu'il y a un certain nombre de cas où ils n'ont rien à dire si je leur dis « on a un arrêté préfectoral qui nous oblige à surveiller la nappe et "ça nous coûte $12 \mathrm{k} \epsilon$ par an », eux ils n'ont rien à dire. Par contre, il y a des cas où l'obligation est loin d'être aussi évidente, ou même si l'obligation est évidente la solution et les options de provisionnement sont ouvertes, donc là le chiffre final qui sera dans nos comptes est vraiment le résultat de cette discussion avec les financiers. »

(Directeur de la réhabilitation environnementale d'un groupe chimique.)

De façon intéressante, le directeur financier du groupe n'a finalement qu'un rôle de validation des montants comptabilisés au titre des provisions environnementales et ne pèse pas fondamentalement sur ces derniers.

\begin{abstract}
« Le niveau de validation que j'évoquais est plutôt le niveau de validation qui vise à prévenir les acteurs centraux, c'est-à-dire le CFO, le contrôleur du groupe. Il va y avoir une évolution de l'estimation, et on les en avertit. Quand je dis validation, c'est plutôt une caractéristique d'information. Bien comprendre le mouvement comptable qui va être inscrit dans les comptes. »
\end{abstract}

(Directeur de la réhabilitation environnementale d'un groupe chimique.)

Les directions des risques interviennent également de façon secondaire pour la quantification des risques, tout en ne traitant pas des provisions directement.

\title{
2.1.2. Acteurs externes influençant le processus de comptabilisation (champ organisationnel)
}

Les premiers acteurs externes à l'entreprise ayant un rôle important dans le contrôle des provisions environnementales comptabilisées sont les commissaires aux comptes. Ils n'interviennent pas dans la décision finale mais sont très au fait des méthodes de calcul utilisées et des hypothèses effectuées. Ils connaissent également bien le processus organisationnel menant à la comptabilisation finale des provisions environnementales. Dans leur mission d'audit, ils peuvent faire (et font) intervenir des spécialistes en environnement pour vérifier la fidélité des provisions environnementales si nécessaire (surtout lorsque les montants sont très matériels) et disposent parfois d'équipes dédiées composées d'ingénieurs et de spécialistes environnement. Les commissaires aux comptes, s'ils n'ont pas un rôle direct dans la comptabilisation des provisions environnementales, ont une influence certaine sur l'issue de leur comptabilisation, acceptant ou non les hypothèses et estimations des groupes. 
Deux autres types d'acteurs influencent encore fortement l'issue du processus de comptabilisation des provisions environnementales, par l'intérêt qu'ils peuvent y porter. Le premier regroupe les administrations publiques environnementales comme les DREAL qui donnent leur accord sur les technologies de dépollution envisagées par les entreprises et donc influencent le montant final des provisions. Le second est l'administration fiscale, qui valide une méthode de calcul des provisions environnementales qui seront alors déductibles. Enfin, un certain nombre d'autres acteurs regardent les provisions environnementales et peuvent potentiellement influencer leur comptabilisation : les analystes financiers, qui posent des questions au sujet des méthodes de calculs, de l'exhaustivité des risques pris en compte, etc. ; les directions comptables des autres groupes d'un même secteur qui sont parfois contactées pour comparaison; l'Autorité des marchés financiers (AMF) qui demande également de plus en plus d'informations sur les méthodes de calculs de ces provisions. La figure 1 synthétise la place des différents acteurs, internes comme externes, dans le processus de comptabilisation des provisions environnementales. 
Figure 1

Cartographie des acteurs impliqués dans le processus de détermination des provisions environnementales

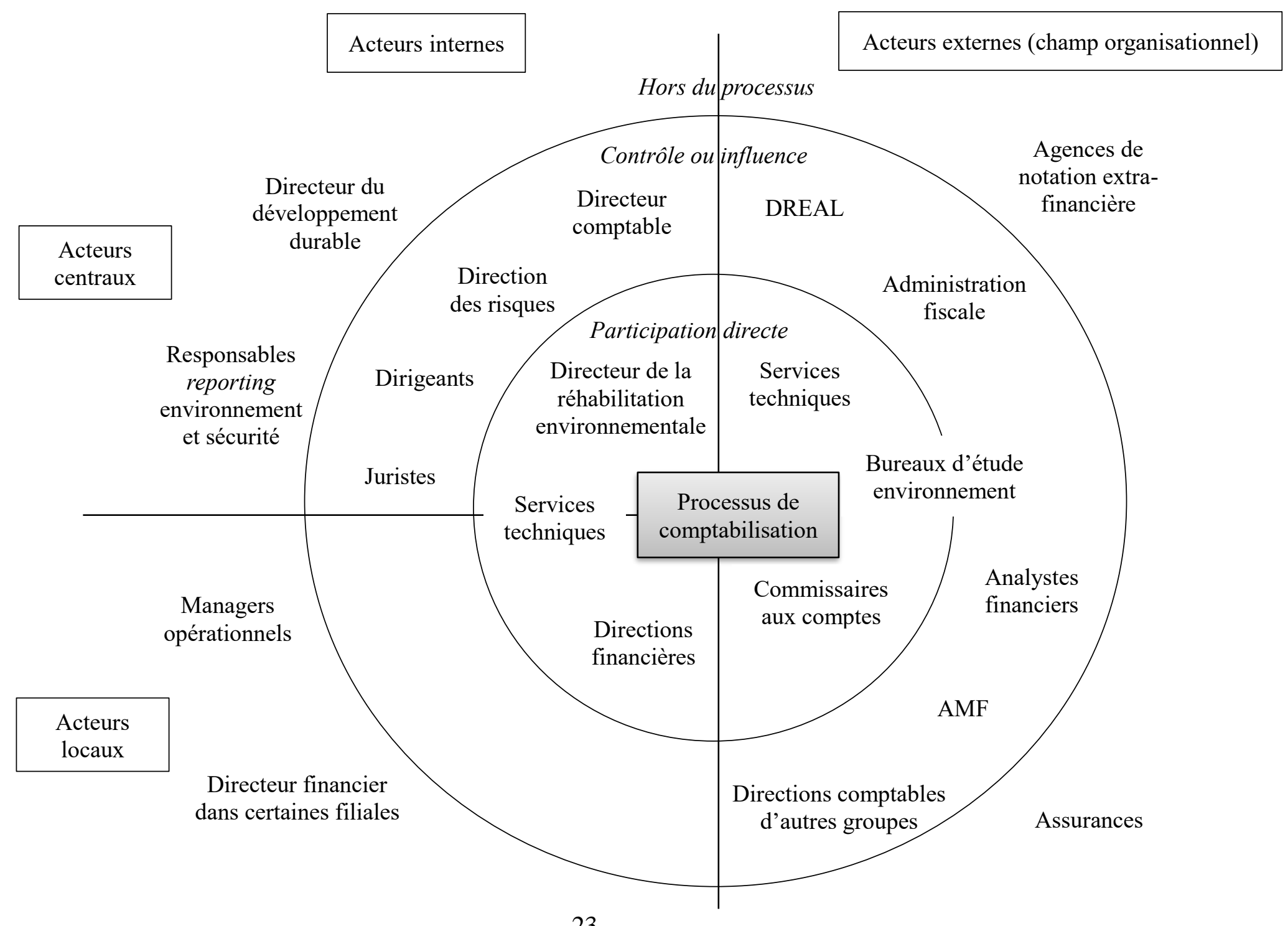




\subsection{Approche institutionnelle : isomorphismes et légitimité}

L'isomorphisme mis en évidence par les entretiens sur le plan de la détermination (estimation et comptabilisation) des provisions environnementales est à la fois coercitif et mimétique (DiMaggio et Powell, 1983). L'aspect normatif ne ressort en revanche pas clairement des entretiens. La recherche de légitimité est enfin un élément important du processus de décision entourant les provisions environnementales.

\subsubsection{Isomorphisme coercitif}

Si les estimations de provisions environnementales sont calculées et validées en interne, différents acteurs viennent encadrer plus ou moins fortement les conditions de leur comptabilisation. Trois acteurs ont ici été nommés à plusieurs reprises par les personnes interrogées comme jouant un rôle important dans la comptabilisation des provisions environnementales. Nous les présentons ici sans hiérarchiser l'importance de leur influence qui peut varier d'une entreprise et d'un secteur à l'autre.

Premièrement, l'administration environnementale française, à travers les directions régionales de l'environnement, de l'aménagement et du logement (DREAL, anciennement DRIRE - directions régionales de l'industrie, de la recherche et de l'environnement), exerce aussi un pouvoir coercitif sur les entreprises, influençant directement les montants comptabilisés en imposant certaines technologies (de dépollution, de sécurité, etc.) aux entreprises.

\footnotetext{
«Tous ces coûts-là font l'objet d'une provision. Et cette provision, qui est définie avec l'administration [les DREAL], va être définie de façon différente selon les techniques de sécurité que vous avez mises en place en amont. »

(Directeur du développement durable d'un groupe de traitement de déchets.)

«Il y a un troisième acteur qui intervient, ce sont les DRIRE. Puisque la plupart du temps, avant par exemple de mettre en cuvre des solutions de dépollution des sols, les entreprises demandent à la DRIRE si ce qui est envisagé les satisfera. Donc souvent, les états financiers peuvent être arrêtés alors que les discussions avec les DRIRE continuent sur une technologie ou une autre, avec des différences de coûts très sensibles. »

(Commissaire aux comptes.)
}

Deuxièmement, l'administration fiscale influence également l'estimation des provisions environnementales comptabilisées en privilégiant une méthode et des hypothèses de calcul pour leur déductibilité, comme l'indique l'un des commissaires aux comptes interrogé :

«Ce type de provisions est déductible et donc généralement le fisc tamponne une méthode. Et une fois qu'il a tamponné une méthode, elle fait le tour de la place et tout le monde utilise la méthode que le fisc a tamponnée. C'est vrai que souvent, on essaye d'avoir les mêmes 
méthodes fiscales et comptables. [...] Ce sont des acteurs qui vont poser des questions, qui vont chercher à comprendre et qui vont avoir tendance à faire en sorte que les méthodes s'uniformisent. »

Troisièmement, le processus d'audit des provisions environnementales conduit les commissaires aux comptes à exercer une influence assez forte lors la revue des provisions environnementales dans les secteurs d'activités où les risques environnementaux sont importants.

«Dans nos métiers, c'est un des sujets que les commissaires aux comptes regardent systématiquement. »

(Directeur comptable et financier d'un groupe d'extraction de minerais.)

"[Revoir] les provisions avec les auditeurs est quelque chose qui prend quand même pas mal de temps. »

(Directeur de la réhabilitation environnementale d'un groupe chimique.)

Cette revue se fait directement avec les ingénieurs environnement des sites, et non avec les directions comptables locales ou centrales, par le biais d'experts environnementaux. La validité des estimations de provisions environnementales est alors questionnée par ces experts de façon approfondie.

«On discute toujours avec les services internes. Il y a plusieurs intervenants. La plupart du temps, il y a les services techniques de la filiale concernée par la provision. Il y a très souvent une sorte de bureau d'études interne ou externe, au niveau central, pour valider ce qui est fait au niveau local. Et puis après, il y a les services financiers, les directions financières qui aujourd'hui essayent de prendre les solutions les moins douteuses. »

(Commissaire aux comptes. Points abordés dans les mêmes termes par les autres personnes interrogées au sein des groupes.)

«Et nous-mêmes, on peut disposer d'experts. On a dans des pays où les enjeux sont importants des équipes qui peuvent être spécialisées et challenger ces provisions-là. C'est notamment les équipes de spécialistes environnementaux dans certains pays. »

(Commissaire aux comptes.)

Dans la mesure où le commissaire aux comptes a un pouvoir de sanction lors du processus d'audit (la certification des comptes avec réserve), l'influence coercitive de celui-ci est prégnante sur l'estimation et la comptabilisation des provisions environnementales lorsqu'elles sont matérielles.

«Le rôle de l'audit, c'est d'émettre un rapport général d'opinion sur les comptes. Ce rapport est soit sans réserve, soit avec réserve. [...] Je me prononce sur les comptes dans leur ensemble et ces provisions-là font partie des comptes. [...] Par exemple, si vous prenez le cas de General Motors, où les fonds de pension de General Motors font le total bilan de General Motors, c'est-à-dire que vous avez autant d'actifs gérés par les fonds de pension de General 
Motors que des actifs General Motors, évidemment que l'audit des retraites de General

Motors c'est aussi important que l'audit de General Motors. »

(Commissaire aux comptes.)

Cette influence coercitive peut d'autant mieux s'exercer que l'auditeur avance dans son mandat avec l'entreprise.

"C'est un travail classique, de s'assurer que les dépenses qui ont été prévues au cours d'une année sont bien celles qui ont été encourues au cours de l'année. Ça c'est sûr. Et de s'assurer de la cohérence des méthodes de calcul de la provision d'une année sur l'autre ou sinon d'avoir des explications sur les raisons pour lesquelles la méthode a été changée ou pourquoi il y a des variations importantes de provisions. Ça c'est la base de nos travaux de contrôle. » (Commissaire aux comptes.)

\title{
2.2.2. Isomorphisme mimétique
}

Il existe également une convergence sectorielle sur les méthodes d'estimation des provisions environnementales, bien que chaque groupe conserve ses spécificités comme l'indique ce commissaire aux comptes :

«Par secteur, vous avez des pratiques, des méthodes de provisionnement. Après, chaque entreprise a ses experts, sa détermination des coûts, ses taux. Vous avez autant de taux d'actualisation de provisions que de provisions. Mais grosso modo ça converge quand même. Je veux dire, vous n'en avez pas un qui est à $10 \%$, l'autre qui est à $2 \%$. [...] Globalement, il y a un travail de convergence fort sur ce type de choses. »

Le degré d'incertitude qui entoure l'évaluation des provisions environnementales conduit les directions comptables à chercher la convergence avec les autres groupes de la même industrie. Les usages professionnels transcendent ici les organisations pour assurer une cohérence des méthodes d'évaluation des risques et coûts environnementaux au sein d'une industrie.

\begin{abstract}
«Enfin moi je m'interroge, en tout cas en tant que comptable, sur la représentation que donne, par exemple, le passif. Elle est peut-être la meilleure estimation d'un flux actualisé de cash-out. Ce que je sais, c'est que c'est un élément qui est extraordinairement variable, suivant les hypothèses que vous prenez. Donc qu'est-ce que vous cherchez à faire quand vous faites face à une forte incertitude comme ça en tant que comptable? Vous essayez de voir si vous arrivez à avoir une vision relativement convergente au sein d'une industrie. [...] On essaye de discuter avec nos pairs pour voir comment ils abordent cette même thématique.

Pour voir si se dégage une approche privilégiée ou un horizon privilégié, etc. »
\end{abstract}

(Directeur des normes comptables d'un groupe de télécommunications.)

D'ailleurs, la comparaison avec les autres groupes permet aux commissaires aux comptes d'évaluer la qualité et la fidélité des estimations du groupe. Il existe alors une certaine convergence 
au sein d'une industrie permettant une meilleure comparabilité des montants comptabilisés. Toutefois, certaines personnes interrogées rappellent que des différences sensibles demeurent d'un groupe à l'autre en termes d'horizon temporel des risques environnementaux (taux d'actualisation) et des provisions, même si certains standards semblent dominer au sein d'une industrie. Car si les groupes regardent ce que font les autres au niveau des provisions environnementales, cela ne semble toutefois pas avoir d'impact sur leurs décisions immédiates en termes de montants comptabilisés (point évoqué par le directeur de la comptabilité d'un groupe automobile, le directeur du développement durable d'un groupe de traitement de déchets et le directeur du développement durable d'un autre groupe automobile). Le processus d'homogénéisation par mimétisme semble donc être un processus relativement lent en comparaison des autres influences institutionnelles.

\begin{abstract}
" [Ça n'a pas trop d'impact sur leurs décisions,] ne serait-ce que parce qu'ils regardent, ils se posent des questions mais ils n'ont pas le détail des calculs, ils n'ont pas le détail des méthodes de calculs, ils n'ont pas les détails site par site, donc ce n'est pas toujours facile de tout comprendre. Mais, bien entendu, ils regardent. Ils regardent l'évolution d'une année sur l'autre. Et pas qu'entre groupes français. »

(Commissaire aux comptes.)
\end{abstract}

\title{
2.2.3. Isomorphisme normatif
}

Les influences normatives sur le processus de détermination des provisions environnementales peuvent venir de la profession comptable ou des spécialistes en environnement (ingénieurs des sites et des bureaux d'études externes). Toutefois, à l'instar de Barbu et Piot (2012) dans le cadre de l'adoption des normes IFRS, ces influences normatives ressortent assez peu des verbatim avec les directeurs comptables interrogés. N'ayant pas eu l'opportunité d'interroger des ingénieurs environnement, il n'est pas possible de se prononcer sur l'influence de leur professionnalisation sur les pratiques d'estimation des provisions. Il est enfin probable que la proximité de formation entre les commissaires aux comptes et les comptables ait une influence normative, mais celle-ci n'est pas ressortie de l'analyse des entretiens.

En synthèse, la figure 2 représente graphiquement les acteurs à l'origine des influences institutionnelles qui tendent à homogénéiser les pratiques d'estimation et de comptabilisation des provisions environnementales en fonction de leur intensité, telles qu'elles ressortent de l'analyse des verbatim.

\subsection{4. À la recherche de légitimité}

Pour expliquer le résultat de ces influences institutionnelles sur les organisations, il est important de mettre en évidence la raison pour laquelle les entreprises s'y soumettent, parfois à l'encontre de toute 
rationalité économique. La recherche de légitimité est pour la théorie néo-institutionnelle une des explications de l'homogénéité des pratiques. Dans notre cas, la recherche d'une légitimité environnementale est partagée par l'ensemble des groupes étudiés, a fortiori lorsque la provision nait d'une obligation implicite, donc non coercitive.

« Si la loi ne vous contraint pas, la dotation d'une provision est plus compliquée parce que vous êtes dans le cas d'une obligation implicite. Donc l'obligation implicite repose sur la contrainte externe qui peut peser sur l'entreprise : soit le risque d'image, soit l'aléa moral, soit une certaine pression d'un environnement politico-environnemental. » (Commissaire aux comptes.)

Pour limiter ces risques qui existent aussi quand l'obligation est explicite, le groupe a intérêt à se montrer légitime à travers ses actions en faveur de l'environnement. C'est pourquoi certains groupes vont au-delà de la réglementation locale de remise en état des sites pour une question d'image, influençant ainsi les montants provisionnés. L'attention portée à l'image du groupe est d'autant plus forte que le groupe est exposé médiatiquement et évolue dans un secteur sensible aux risques environnementaux, notamment sur les incidents environnementaux qu'il peut subir (élément abordé par les groupes pétrolier et pharmaceutique étudiés).

«Quand une initiative est prise pour aller au-delà des réglementations, c'est que nous en faisons un sujet d'image. »

(Directeur de la comptabilité d'un groupe automobile.)

Enfin, le fait d'aller au-delà de la réglementation environnementale locale reste un moyen largement utilisé pour se légitimer (amélioration de l'image, affichage de la bonne volonté du groupe sur le sujet environnemental, etc.) :

«On fait des mesures volontaristes par rapport à notre secteur. [...] Tout ce qu'on fait c'est en plus [...] pour montrer qu'on a une bonne volonté sur le sujet. »

(Directeur hygiène, sécurité et environnement d'un groupe de télécommunications.) 


\section{Figure 2}

Acteurs influençant les pratiques d'estimation et comptabilisation des provisions environnementales

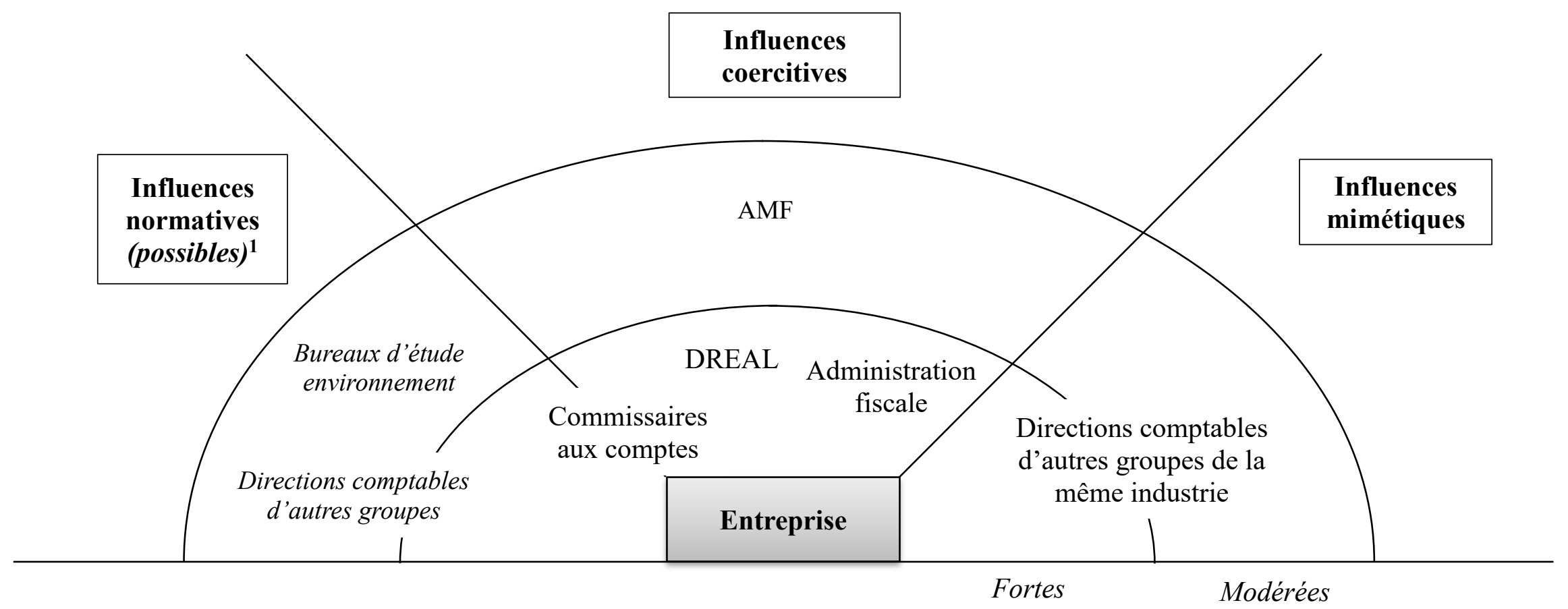

1. Les influences normatives possibles ne sont pas ressorties clairement des entretiens. Elles sont indiquées en italique. 


\subsection{Approche par la théorie positive : une gestion du résultat sous contraintes}

\subsubsection{Une gestion du résultat possible mais limitée}

À la lumière des entretiens effectués avec différents acteurs du processus dans plusieurs organisations, y compris des commissaires aux comptes, il n'est pas évident de valider la proposition selon laquelle les provisions environnementales, en particulier celles de remise en état de sites, servent à gérer le résultat comptable. En effet, il ressort que les provisions pour remise en état de sites ne sont pas un élément de pilotage, mais sont avant tout un élément d'activité. Elles semblent évaluées sérieusement par des experts au sein d'une organisation qualifiée de «professionnelle » par l'un des commissaires aux comptes interrogés.

\footnotetext{
«Mon impression, c'est qu'à partir du moment où c'est dans le coût de revient de votre prestation [...], c'est trop dangereux de traiter ça de façon non industrielle ou non professionnelle. C'est-à-dire, c'est un peu comme si vous me dites dans les compagnies d'assurance, qui est-ce qui fait les provisions? Est-ce que c'est le directeur financier ou estce que c'est le service d'actuariat où il y a cinquante actuaires en batterie qui sont chargés de calculer le risque auto avec des tables hyper savantes? À partir du moment où votre business c'est ça, où le business d'EDF c'est de gérer son risque nucléaire et y compris le risque de démantèlement, et où le business de Veolia, c'est de gérer ses décharges, y compris le risque de devoir la porter pendant cinquante ans après sa fermeture, et bien vous avez une organisation professionnelle derrière et industrielle avec des experts. Ce n'est pas un élément de pilotage. C'est un élément de business. C'est un élément de votre coût de revient. C'està-dire si vous n'estimez pas bien votre coût de revient, vous ne tarifez pas bien. Je pense que la comparaison avec l'assurance est bonne dans le sens où vous avez les revenus avant d'avoir les charges. »

(Commissaire aux comptes.)
}

Elles pourraient être surestimées mais ce ne serait pas vraiment dans l'intérêt des groupes, d'autant que l'administration fiscale est assez vigilante in fine. Il y a toutefois une possibilité de limiter les provisions pour remise en état de sites par un surinvestissement en sécurité au fur et à mesure. Les travaux effectués au fil du temps permettent de réduire le montant des travaux à réaliser lors de la fermeture du site, et donc la provision, ce qui est une façon de reporter des profits dans le futur. Les provisions pour remise en état de sites sont des charges à part entière qui sont répercutées sur le coût de production et donc sur les clients (lorsqu'elles sont significatives, elles font généralement partie du business model du groupe).

«Lorsque vous avez une obligation de remise en état, ça fait partie de vos coûts de production. C'est-à-dire qu'EDF va prendre en compte et a toujours pris en compte dans le coût de son kilowattheure, le fait qu'elle avait des centrales à remettre en état. Quand Veolia 
facture une tonne de déchets à l'enfouissement, le prix que les gens payent pour aller enfouir leurs déchets prend en compte le fait qu'il va falloir remettre la décharge en état. [...] Ça a toujours pesé sur les comptes et ça pèsera toujours sur les comptes, parce que ça fait partie du business model de ces boites-là. C'est une charge à part entière. »

(Commissaire aux comptes.)

Ce point justifie l'intérêt pour l'entreprise de ne pas se tromper dans ses évaluations et de ne pas prendre de risques en sous-estimant la provision (voir la prudence des directions financières centrales évoquée ci-dessus). L'enjeu de ne pas se tromper est donc d'abord un enjeu de tarification, mais également un enjeu légal puisqu'une erreur matérielle dans la comptabilisation des provisions place le groupe dans l'illégalité par rapport à ses précédentes déclarations de résultat.

Toutefois, le mécanisme comptable des provisions pour remise en état de sites fait qu'il y a des effets mécaniques induits qui les rendent contracycliques : une réduction de l'activité entraîne un report dans le temps de la charge de remise en état (dotée en proportion de l'activité, de la même façon que l'amortissement des actifs liés). Du fait de l'actualisation, cela implique une réduction de la charge globale (la charge actuelle devient plus faible). L'impact sur le résultat est alors le suivant : la baisse de l'activité entraîne une baisse de la dotation (voire une reprise en raison de la baisse de la charge restante actualisée) et inversement. Toutes choses égales par ailleurs, il est donc logique d'observer une corrélation positive entre le résultat comptable et les dotations aux provisions pour remise en état de sites, sans que celle-ci puisse être considérée comme le signe d'une gestion du résultat (par lissage). C'est le cas pour les entreprises d'enfouissement de déchets mais aussi dès lors que les provisions environnementales requièrent l'amortissement d'un actif dédié à un rythme suivant la détérioration réelle du site.

«Ce n'est pas qu'ils ont tripatouillés leurs comptes. Mais, c'est qu'en fait vous avez un effet de ralentissement du remplissage des décharges et mécaniquement vous faites vos dépenses plus tard, et donc mécaniquement la valeur actualisée de ces dépenses est moins [élevée]. [...] Donc en fait, l'évolution de ces provisions peut se retrouver un moment contracyclique, pas par pilotage de résultat mais juste dans leur mode de calcul. »

(Commissaire aux comptes.)

L'explication du lissage du résultat dans le cas des provisions pour remise en état de sites des entreprises canadiennes (Berthelot et al., 2003) pourrait alors en fait être non valide dans le cas français $^{8}$. Pour estimer la gestion du résultat, il est plus pertinent d'étudier les reprises sans objet,

8. Une réplication de l'étude quantitative de Berthelot et $a l$. (2003) a été effectuée sur les groupes cotés français publiant le montant de leurs provisions environnementales pour la période 2005-2010 (période précédant celle des entretiens afin de conserver la même période d'analyse). Contrairement à l'étude de Berthelot et al. (2003) sur les entreprises canadiennes, les résultats (non présentés dans cet article) indiquent que les provisions environnementales 
même si elles peuvent se justifier dans plusieurs cas (évolution réglementaire, fin d'un litige, évolution technologique, revenus complémentaires liés à la remise en état [valorisation des déchets, etc.]) et donc être légitimes.

Pour le second commissaire aux comptes interrogé, il est toutefois évident que les provisions sont une variable d'ajustement pour les groupes, a fortiori pour les provisions environnementales ne donnant pas lieu à la constitution d'un actif qui laissent une bonne marge de manœuvre et concernent des montants souvent significatifs.

«Vous ne le répèterez pas parce que c'est bien évident [que les provisions environnementales peuvent être utilisées pour lisser le résultat]. Les années où les résultats sont bons, il est beaucoup plus facile de passer des provisions que les années où les résultats sont mauvais. [...] sur des montants qui peuvent être plus significatifs que sur un litige prudhommal ou des choses comme ça.»

(Commissaire aux comptes.)

Ce risque semble pourtant modéré par la taille de l'entreprise et la pression politique qu'elle subit. Ainsi, plus le groupe est important, moins ces comportements de gestion des données comptables semblent se manifester selon le même commissaire aux comptes.

«Alors ça quand même, les grands groupes ne jouent pas beaucoup avec ça. Les plus petits groupes, oui, c'est quelque chose qui est plus utilisé. »

(Commissaire aux comptes.)

Finalement, il ressort des entretiens avec les commissaires aux comptes que les provisions environnementales pour remise en état de sites seraient beaucoup moins sujettes à manipulation que les provisions pour risques environnementaux qui ne donnent pas lieu à la constitution d'un actif. De plus, cette possibilité de manipulation semble réduite pour les entreprises de grandes tailles, donc visibles, qui cherchent à maintenir leur légitimité et éviter des coûts politiques en constatant un niveau crédible de provisions environnementales. La portée de cette conclusion doit toutefois être mesurée au regard des limites présentées par l'approche qualitative. La réalité d'une gestion du résultat est en effet difficilement décelable par des entretiens avec les acteurs eux-mêmes (qui auraient tendance à ne pas en parler ou pourraient ne pas en être informés).

comptabilisées par les groupes cotés français ne sont pas utilisées pour lisser le résultat comptable ni pour éviter les coûts politiques liés à la promulgation de la loi Grenelle 2 sur la période d'analyse. Ces résultats vont dans le sens d'une certaine prudence à considérer que ces provisions sont utilisées pour gérer le résultat comptable. 


\subsubsection{Des choix comptables contraints par les techniques d'évaluation}

Les entretiens réalisés avec différents acteurs internes et externes du processus d'évaluation des provisions environnementales révèlent enfin que les montants comptabilisés in fine dépendent de la façon dont les ingénieurs environnement des sites réalisent les premières estimations de coûts. Le processus de détermination des provisions environnementales est donc centré sur les évaluations techniques des sites qui ne sont pas faites par des comptables. Ces derniers ont donc peu de marge de manœuvre pour modifier les premières estimations des sites dans une perspective de gestion du résultat. Par exemple, concernant les provisions de remise en état des décharges, un commissaire aux comptes indique que :
«Ce sont des provisions qui sont revues avec les services techniques. C'est-à-dire des provisions qui sont souvent préparées par ces services techniques... Ça fait partie des provisions les plus techniques avec les provisions pour retraite. Ce sont des services qui sont généralement indépendants d'un certain nombre de donneurs d'ordre opérationnels. [...] Dans les sociétés, il y a une problématique de ségrégation des tâches qui est assez forte sur ces sujets-là parce que vous avez des experts techniques qui sont souvent indépendants des managers opérationnels. Les managers opérationnels doivent gérer en fonction des impératifs techniques qui leur sont donnés par ces gens-là. »

En interne, le processus de décision semble tout de même partagé entre les fonctions techniques et financières à partir des premières estimations réalisées par les sites. Le directeur de la réhabilitation environnementale du groupe chimique interrogé indique dans ce sens que :

\footnotetext{
«La première étape du processus, c'est de discuter avec eux [les services techniques des sites] sur un plan technique. Et cette discussion technique, qui est tout à fait ouverte, aboutit à l'évaluation d'une sortie de cash-flows. [...] On regarde s'il y a une obligation, on regarde s'il y a une sortie probable de cash et c'est cette sortie probable de cash qu'on discute avec les sites pour savoir effectivement quel peut être le cash-flow envisageable. Et donc c'est l'ensemble de ces cash-flows que je remonte moi, enfin que je collecte, que je centralise et que je discute après avec la fonction corporate. Il y a quand même un certain nombre de cas où la décision est aussi bien technique que financière en matière de provision. »
}

Pour autant, la marge de manœuvre des comptables reste relativement limitée dans la décision finale comme l'évoque le directeur environnement d'un groupe pharmaceutique :

« Alors, clairement l'expert environnement, sur les provisions, est le plus à même d'apprécier les choses. Donc s'il sort un chiffre, le juridique ou le financier va pas dire " mais ce chiffrelà je ne le comprends pas. » 
Enfin, la formation des personnes chargées de la détermination des provisions environnementales est essentiellement une formation d'ingénieur, limitant de fait l'influence des comptables dans la détermination des cash-flows sur lesquels se basent les provisions :

«Je dirais que [la formation de mon équipe] est essentiellement ingénieur. Alors pas forcément des ingénieurs environnement mais ce sont des gens qui ont une certaine expérience quand même des sites ou qui ont une grosse formation effectivement en matière d'environnement. »

(Directeur de la réhabilitation environnementale du groupe chimique étudié.)

Le caractère technique et calculatoire des provisions environnementales pour remise en état de site pourrait donc limiter l'usage discrétionnaire qui peut être fait de ce type de provisions.

\subsection{Discussion}

En résumé, l'analyse des entretiens permet de mettre en évidence la complémentarité possible entre les deux approches théoriques dans la compréhension des choix comptables relatifs aux provisions environnementales. Le tableau 1 synthétise les résultats par cadre théorique et type de provisions environnementales en distinguant la phase d'estimation des coûts (par les experts environnement) de la phase de comptabilisation des provisions (par les directions comptables).

\section{Tableau 1}

Synthèse des résultats par approche théorique et type de provisions environnementales

\begin{tabular}{|c|c|c|c|c|}
\hline & \multicolumn{2}{|c|}{$\begin{array}{l}\text { Provisions pour remise en état de site } \\
\text { (dépenses activées) }\end{array}$} & \multicolumn{2}{|c|}{$\begin{array}{l}\text { Provision pour risques environ. } \\
\text { (dépenses non activées) }\end{array}$} \\
\hline & Estimation & Comptabilisation & Estimation & Comptabilisation \\
\hline $\begin{array}{l}\text { Approche néo- } \\
\text { institutionnelle }\end{array}$ & $\begin{array}{l}\text { - Influence coercitive } \\
\text { des DREAL et des } \\
\text { commissaires aux } \\
\text { comptes } \\
\text { - Influence } \\
\text { mimétique des autres } \\
\text { groupes du même } \\
\text { secteur }\end{array}$ & $\begin{array}{l}\text { - Influence coercitive } \\
\text { de l'administration } \\
\text { fiscale et des } \\
\text { commissaires aux } \\
\text { comptes } \\
\text { - Influence mimétique } \\
\text { des autres groupes du } \\
\text { même secteur } \\
\text { - Recherche de } \\
\text { légitimité par la } \\
\text { comptabilisation de } \\
\text { provisions issues } \\
\text { d'obligations } \\
\text { implicites }\end{array}$ & $\begin{array}{l}\text { - Influence } \\
\text { coercitive des } \\
\text { DREAL et des } \\
\text { commissaires aux } \\
\text { comptes } \\
\text { - Influence } \\
\text { mimétique des } \\
\text { autres groupes du } \\
\text { même secteur }\end{array}$ & $\begin{array}{l}\text { - Influence } \\
\text { coercitive de } \\
\text { l'administration } \\
\text { fiscale et des } \\
\text { commissaires aux } \\
\text { comptes }\end{array}$ \\
\hline
\end{tabular}




\begin{tabular}{|c|c|c|c|}
\hline $\begin{array}{l}\text { Approche de la } \\
\text { théorie positive }\end{array}$ & $\begin{array}{l}\text { - Manipulation } \\
\text { limitée car la forte } \\
\text { technicité est entre les } \\
\text { mains des ingénieurs } \\
\text { environnement et non } \\
\text { des comptables }\end{array}$ & $\begin{array}{l}\text { - Manipulation limitée } \\
\text { car intérêt à intégrer } \\
\text { fidèlement les coûts de } \\
\text { démantèlement dans } \\
\text { les coûts de revient }\end{array}$ & $\begin{array}{l}\text { - Manipulation possible, surtout pour les } \\
\text { entreprises peu visibles : montants } \\
\text { importants parfois difficiles à auditer }\end{array}$ \\
\hline
\end{tabular}

L'analyse des résultats peut se faire à deux niveaux, celui des acteurs et celui de l'organisation, tant pour expliciter la difficulté de la théorie positive comptable à expliquer totalement les comportements de détermination des provisions environnementales que pour analyser les comportements isomorphes mis en évidence. Il convient de noter que ces résultats et analyses ne concernent que les groupes cotés suffisamment grands pour subir des risques environnementaux devant entrainer la comptabilisation de provisions environnementales significatives, et la diffusion de leur montant dans le document de référence. Comme l'a rappelé l'un des commissaires aux comptes interrogés (voir le dernier verbatim de la section 2.3.1), les problématiques de gestion du résultat à partir des provisions environnementales sont différentes pour les petits groupes moins visibles.

Au niveau des acteurs dans l'organisation (et en particulier des dirigeants), plusieurs éléments peuvent expliquer qu'ils ne poursuivent pas uniquement leur intérêt personnel dans la comptabilisation des provisions environnementales. Tout d'abord, la dimension technique de l'évaluation des risques environnementaux donne un poids certain aux acteurs locaux qui possèdent la compétence. La connaissance de l'expert (ingénieur des services techniques) face à l'ignorance du manager entraîne de fait un partage du pouvoir de décision dans la comptabilisation des provisions environnementales. Les services techniques locaux, s'ils doivent reporter l'information à la maisonmère, n'ont en effet pas les mêmes intérêts que les dirigeants, ni la même logique de fonctionnement (confrontation de la logique de l'ingénieur et de celle du manager). Par ailleurs, l'incertitude entourant l'évaluation des coûts environnementaux ne peut que limiter les comportements d'optimisation de la part des dirigeants dans une perspective de rationalité limitée. Les entretiens révèlent en effet que cette incertitude conduit le comptable à rechercher des solutions convergentes au sein d'une même industrie et interroge la représentation comptable de leur entreprise que donnent de telles estimations. Par conséquent, les solutions retenues sont davantage des solutions acceptables (par les dirigeants, par les ingénieurs producteurs de l'information brute, par les commissaires aux comptes, par l'administration fiscale, par les DREAL) que des solutions optimales (du point de vue des dirigeants seulement).

Au niveau de l'organisation, les entretiens révèlent l'influence d'un certain nombre de pressions institutionnelles dans l'issue du processus de détermination des provisions environnementales. Ces 
pressions institutionnelles sont diverses et se complètent généralement. Par exemple, sur le plan comptable, l'administration fiscale va considérer certaines méthodes de comptabilisation comme préférentielles selon sa propre logique. Elle contribue ainsi à l'alignement des méthodes comptables sur les méthodes fiscales, ce qui réduit de fait les marges de manœuvre à disposition des dirigeants dans la comptabilisation des provisions environnementales. Sur le plan technique, les DREAL vont apporter elles aussi leurs exigences en ce qui concerne le choix des technologies de dépollution envisagées. Deux technologies différentes pouvant entraîner des coûts significativement différents, l'influence des DREAL est donc directe sur les montants comptabilisés au titre des provisions pour remise en état de sites et constitue une limitation immédiate des marges de manœuvre des dirigeants. Enfin, en dernier lieu, les commissaires aux comptes analysent et valident les méthodes de calcul et les hypothèses retenues en travaillant directement avec les services techniques. Ils évaluent donc directement le caractère raisonnable des montants comptabilisés au titre des provisions environnementales. Ces trois acteurs à l'origine d'influences coercitives constituent autant de limites pratiques aux marges de manœuvre concédées aux dirigeants en matière de provisions environnementales. L'organisation devant répondre à de nombreuses demandes institutionnelles en matière de provisions environnementales, l'hypothèse de la recherche par les dirigeants de leur intérêt personnel à travers leur comptabilisation ne peut être complètement invalidée mais sa réalisation en est clairement limitée.

En ce qui concerne les pratiques de détermination des provisions environnementales, l'isomorphisme mimétique mis en évidence dans les résultats peut également s'expliquer sur les deux niveaux d'analyse organisation et acteurs. La première explication, d'ordre macro-économique, est celle classiquement avancée par la théorie néo-institutionnelle : les organisations tendent à adopter les pratiques des autres organisations déjà perçues comme légitimes par l'ensemble des parties prenantes (dont elles dépendent pour leur survie à long terme). Cette explication suppose que les acteurs au sein des organisations savent reconnaître ces pratiques « légitimantes » et les adoptent dans le but d'insérer l'organisation dans la sphère d'activités légitimes aux yeux de l'extérieur. Les entretiens conduits avec les comptables participant à la détermination des provisions environnementales permettent d'affiner cette explication institutionnelle par un ancrage plus microéconomique. Les pratiques de place qui se développent au niveau de la comptabilisation des provisions environnementales semblent initialement liées à l'incertitude à laquelle les comptables font face lors de la comptabilisation de ces provisions. Celle-ci les conduit à rechercher des solutions existantes et, effectivement, déjà acceptées par les parties prenantes externes intéressées par ces provisions (commissaires aux comptes, DREAL, administration fiscale, analystes financiers, 
investisseurs). L'incertitude vécue individuellement par les acteurs renforce donc la pression coercitive exercée par ces dernières. Mimer les autres entreprises a deux implications : au niveau des acteurs, cela réduit l'inconfort lié à l'incertitude de la prospective comptable et, au niveau de l'organisation, cela légitime ses choix vis-à-vis des parties prenantes exerçant sur elle une pression coercitive. L'influence de ces parties prenantes indique que l'isomorphisme mimétique est directement lié, dans le cas des provisions environnementales, à l'isomorphisme coercitif impliqué par ces dernières. La pression institutionnelle coercitive des commissaires aux comptes, des DREAL et de l'administration fiscale, combinée à une forte part d'incertitude dans la comptabilisation des provisions environnementales, conduit les comptables des entreprises d'un même secteur à adopter des pratiques et des hypothèses similaires.

Si l'isomorphisme coercitif semble ainsi dominer le processus, et bien que les résultats n'aient pas pu en faire état, il reste possible d'émettre une dernière hypothèse sur l'existence de pratiques convergentes. La dimension technique de l'estimation des provisions environnementales requiert des formations pointues de niveau ingénieur et la question de l'isomorphisme normatif au niveau des ingénieurs environnement reste ouverte. Les normes professionnelles de ce métier en développement sont en effet susceptibles de conduire les services techniques des entreprises à des solutions et estimations plus ou moins similaires, ce qui reste à étudier en interrogeant ces acteurs dans de futures recherches.

Enfin, le processus de détermination des provisions environnementales repose, dans la plupart des groupes étudiés, sur des rites organisationnels relativement formalisés. Les discussions entre la direction centrale et les services techniques des sites relèvent de ces rites organisationnels lorsqu'ils sont réguliers (cas par exemple du groupe chimique étudié). Ces rites sont malgré tout rationalisés sur le plan de la forme (réunions téléphoniques, fiches techniques à remplir à échéances précises, etc.) même si parfois, ils peuvent être davantage informels (comme cela semble le cas pour le groupe d'extraction de minerais étudié). Ce processus comptable requiert en tous les cas un dialogue entre le monde ingénieur qui détermine les sorties de ressources prévues et le monde comptable qui les accepte et les transforme en provisions.

Ces résultats viennent illustrer la conclusion de Barbu et al. (2014) montrant que les pratiques de comptabilisation et de diffusion des informations environnementales obligatoires en normes IASIFRS (les provisions en constituant une grande part) sont très différentes d'un pays à l'autre. Ils en concluent que la variété des pressions institutionnelles (réglementation, habitudes de reporting, attentes des marchés financiers), plus que les normes comptables, orientent les comportements. L'approche qualitative mise en œuvre dans cette étude a donc permis de déterminer plus précisément 
la nature de ces pressions institutionnelles et de détailler leur influence sur les pratiques de comptabilisation des provisions environnementales.

\section{Conclusion}

Cet article contribue au débat sur l'utilisation discrétionnaire de données comptables à caractère environnemental par les dirigeants en approfondissant l'analyse du processus de décision attaché à la comptabilisation des provisions environnementales par une analyse qualitative. Cette dernière révèle ainsi que les comportements opportunistes, s'ils peuvent exister, ne sont pas aussi déterminants dans le choix des montants comptabilisés in fine. L'analyse des entretiens menés soutient davantage une lecture institutionnelle des comportements menant à la comptabilisation des provisions environnementales (isomorphisme mimétique en présence d'incertitude ; isomorphisme coercitif par, entre autre, l'influence forte des commissaires aux comptes, des DREAL et de l'administration fiscale), qu'une lecture issue la théorie positive comptable (pas d'influence forte des dirigeants ou du directeur financier du groupe ; peu de place laissée à la gestion du résultat).

Les contributions de l'article sont doubles. Sur le plan théorique, tout d'abord, nous prolongeons les travaux de Neu et Simmons (1996) en mobilisant la théorie néo-institutionnelle pour documenter un processus de détermination d'accruals, traditionnellement envisagé sous la seule perspective de la théorie positive. Les résultats permettent de révéler la complexité sociale dans laquelle sont prises les décisions comptables entourant les provisions environnementales, ne pouvant réduire leur détermination à de simples calculs rationnels de la part des dirigeants. En cartographiant la variété d'acteurs pouvant influencer la décision finale de provisionner un risque ou un démantèlement de site, nous montrons que cette dernière est le fruit d'une longue décision collective issue de nombreux compromis, tant entre acteurs internes qu'avec l'extérieur de l'organisation. Les résultats rappellent que la comptabilité ne fait que refléter l'impact financier d'événements et d'opérations économiques ou techniques qui sont en majorité conclus, négociés ou évalués par des professionnels non comptables. Les marges de manœuvre entourant la mesure comptable de ces événements et opérations est alors réduite par les interactions de plusieurs mondes professionnels. Sans rejeter l'idée d'une utilisation opportuniste des provisions environnementales, les résultats suggèrent l'importance de prendre en compte d'autres facteurs d'influence, comme ceux mis en évidence par la théorie néoinstitutionnelle. Sur le plan méthodologique, enfin, l'apport principal de cette étude repose sur le recours à une approche qualitative pour comprendre et faire émerger la façon dont les acteurs 
conçoivent la détermination des provisions environnementales. Le croisement des points de vue entre comptables, directeurs risques, directeurs développement durable et commissaires aux comptes a permis de mettre en lumière leurs rôles (ou non rôles) dans le processus, là où une approche quantitative (par les modèles d'accruals par exemple) ne permet que de supposer des comportements à partir des nombres comptables.

Malgré ces apports, notre étude présente plusieurs limites. Tout d'abord, si l'étude de cas multiple menée ici présente l'avantage d'améliorer la portée des résultats, elle n'a pas permis d'étudier l'intégralité du processus de décision au sein d'une organisation. Une première perspective de recherche est de conduire une étude de cas approfondie des relations entre les acteurs d'une même organisation lorsqu'ils traitent de l'estimation et de la comptabilisation des provisions environnementales. En allant notamment interroger les services techniques des sites et leurs responsables qui sont des parties prenantes importantes du processus de détermination des provisions environnementales, il sera possible de mettre en évidence, par exemple, les éventuels conflits qu'ils peuvent avoir avec la direction centrale. De plus, l'étude ayant montré que les DREAL et l'administration fiscale influençaient les montants de provisions environnementales comptabilisés, il conviendrait d'interroger également ces acteurs.

Ensuite, la question de l'isomorphisme normatif au niveau des ingénieurs environnement mérite d'être approfondie dans la mesure où il n'a pas pu être clairement mis en évidence dans cette étude. La question de l'influence des normes professionnelles sur les méthodes de calcul retenues par les services techniques pour évaluer les coûts de dépollution futurs, en fonction des technologies envisagées, mériterait une étude approfondie. Par ailleurs, la question de la gestion du résultat n'est pas traitée ici de façon complète dans la mesure où les entretiens effectués restent déclaratifs, et que le sujet est délicat à aborder avec les répondants. Une approche quantitative pourrait venir compléter la présente analyse afin d'envisager cette question en prenant en compte les effets contracycliques des provisions pour remise en état de site mis en évidence grâce aux entretiens. Enfin, les conclusions de cette étude sont limitées à l'espace-temps dans lequel elle a été réalisée, à savoir aux grands groupes cotés français avant la promulgation de la loi Grenelle 2, sachant que ce changement réglementaire n'a pas affecté les règles de comptabilisation des provisions. Cette limite appelle à poursuivre aujourd'hui l'analyse sur les grands groupes pour déceler d'éventuels changements dans les méthodes de comptabilisation et dans les facteurs institutionnels influençant les décisions de provisionnement, et à l'étendre à des groupes de taille plus modeste dont les pratiques peuvent être différentes. Cette limite ne remet toutefois pas en cause les contributions de la présente étude qui 
visait à mettre en évidence l'importance et l'influence des facteurs institutionnels sur les choix comptables relatifs aux accruals.

Les provisions environnementales étant amenées à croître dans les années qui viennent en raison de l'importance croissante des réglementations environnementales, cette étude caractérise en première exploration les enjeux entourant l'estimation et la comptabilisation des impacts environnementaux des activités industrielles, tant pour les auditeurs que pour les investisseurs.

\section{Bibliographie}

Baboukardos D. (2017), « The Valuation Relevance of Environmental Performance Revisited: The Moderating Role of Environmental Provisions », British Accounting Review, sous presse.

Barbu E.M. (2006), « Les entreprises françaises cotées face à l'harmonisation comptable internationale : une analyse néo-institutionnelle d'un long processus vers l'homogénéité », thèse de doctorat en sciences de gestion, université d'Orléans.

Barbu E.M., Dumontier P., Feleagǎ N. et Feleagă L. (2014), « Mandatory Environmental Disclosures by Companies Complying with IASs/IFRSs: The Cases of France, Germany, and the UK », International Journal of Accounting, vol. 49, nº 2, p. 231-247.

Barbu E.M. et Piot C. (2012), «L'adoption des IAS/IFRS par les groupes français cotés », Revue Française de Gestion, vol. 38, n 226, p. 53-74.

Barth M.E. et McNichols M.F. (1994), « Estimation and Market Valuation of Environmental Liabilities Relating to Superfund Sites », Journal of Accounting Research, vol. 32, supplément, p. 177-210.

Berthelot S., Cormier D. et Magnan M. (2003), « Les provisions environnementales et la gestion stratégique des résultats : une étude canadienne », Comptabilité - Contrôle-Audit, tome 9, vol. 2, p. 109-135.

Bewley K. (2005), « The Impact of Financial Reporting Regulation on the Market Valuation of Reported Environmental Liabilities: Preliminary Evidence from US and Canadian Public Companies », Journal of International Financial Management and Accounting, vol. 16, $\mathrm{n}^{\mathrm{o}} 1$, p. 1-48.

Boland L.A. et Gordon I.M. (1992), « Criticizing Positive Accounting Theory », Contemporary Accounting Research, vol. 9, $\mathrm{n}^{\circ}$ 1, p. 142-170.

Campbell K., Sefcik S.E. et Soderstrom N.S. (2003), « Disclosure of Private Information and Reduction of Uncertainty: Environmental Liabilities in the Chemical Industry », Review of Quantitative Finance and Accounting, vol. 21, n 4 , p. 349-378.

Clarkson P.M., Li Y. et Richardson G.D. (2004), « The Market Valuation of Environmental Capital Expenditures by Pulp and Paper Companies », Accounting Review, vol. 79, nº 2, p. 329-353.

DiMaggio P.J. (1988), « Interest and Agency in Institutional Theory », in L.G. Zucker (éd.), Institutional Patterns and Organizations: Culture and Environment, Ballinger Pub. Co.

DiMaggio P.J. et Powell W.W. (1983), « The Iron Cage Revisited: Institutional Isomorphism and 
Collective Rationality in Organizational Fields », American Sociological Review, vol. 48, $\mathrm{n}^{0} 2$, p. 147-160.

Farrelly G.E., Ferris K.R. et Reichenstein W.R. (1985), « Perceived Risk, Market Risk, and Accounting Determined Risk Measures », Accounting Review, vol. 60, n 2, p. 278-288.

Hall S.C. et Stammerjohan W.W. (1997), « Damage Awards and Earnings Management in the Oil Industry », Accounting Review, vol. 72, n 1, p. 47-65.

Han J.C.Y. et Wang S. (1998), « Political Costs and Earnings Management of Oil Companies During the 1990 Persian Gulf Crisis », Accounting Review, vol. 73, nº 1, p. 103-117.

Jensen M.C. et Meckling W.H. (1976), « Theory of the Firm: Managerial Behavior, Agency Costs and Ownership Structure », Journal of Financial Economics, vol. 3, n ${ }^{\circ}$ 4, p. 305-360.

Johnston D. et Rock S. (2005), «Eamings Management to Minimize Superfund Clean-up and Transaction Costs », Contemporary Accounting Research, vol. 22, nº 3, p. 617-642.

Lev B. et Kunitzky S. (1974), « On the Association Between Smoothing Measures and the Risk of Common Stocks », Accounting Review, vol. 49, n² 2, p. 259-270.

Li Y. et McConomy B.J. (1999), « An Empirical Examination of Factors Affecting the Timing of Environmental Accounting Standard Adoption and the Impact on Corporate Valuation », Journal of Accounting, Auditing \& Finance, vol. 14, nº 3, p. 279-313.

Meyer J.W. et Rowan B. (1977), « Institutionalized Organizations: Formal Structure as Myth and Ceremony », American Journal of Sociology, vol. 83, n 2, p. 340-363.

Mezias S.J. (1990), «An Institutional Model of Organizational Practice: Financial Reporting at the Fortune 200 », Administrative Science Quarterly, vol. 35, nº 3, p. 431-457.

Miles M.B. et Huberman M. (1984), Qualitative Data Analysis: A Sourcebook of New Methods, Sage Publications.

Milne M.J. (2002), « Positive Accounting Theory, Political Costs and Social Disclosure Analyses: A Critical Look », Critical Perspectives on Accounting, vol. 13, $\mathrm{n}^{\circ}$ 3, p. 369-395.

Mouck T. (1990), « Positive Accounting Theory as a Lakatosian Research Programme », Accounting and Business Research, vol. 20, nº 79, p. 231-239.

Negash M. (2012), «IFRS and Environmental Accounting », Management Research Review, vol. $35, \mathrm{n}^{\mathrm{0}} 7$, p. $577-601$.

Neu D. (1992), « The Social Construction of Positive Choices », Accounting, Organizations and Society, vol. 17, $\mathrm{n}^{\text {os }} 3-4$, p. 223-237.

Neu D. et Simmons C. (1996), «Reconsidering the "Social" in Positive Accounting Theory: The Case of Site Restoration Costs », Critical Perspectives on Accounting, vol. 7, $\mathrm{n}^{\circ}$ 4, p. 409-435.

Patten D.M. et Trompeter G. (2003), « Corporate Responses to Political Costs: An Examination of the Relation Between Environmental Disclosure and Earnings Management », Journal of Accounting and Public Policy, vol. 22, $\mathrm{n}^{\mathrm{0}}$ 1, p. 83-94.

Peek E. (2004), « The Use of Discretionary Provisions in Earnings Management: Evidence from The Netherlands », Journal of International Accounting Research, vol. 3, nº 2, p. 27-43.

Posner R.A. (1974), « Theories of Economic Regulation », Bell Journal of Economics and Management Science, vol. 5, $\mathrm{n}^{\circ}$ 2, p. 335-358.

Schneider T., Michelon G. et Maier M. (2017), « Environmental Liabilities and Diversity in Practice 
Under International Financial Reporting Standards », Accounting, Auditing \& Accountability Journal, vol. 30, no 2, p. 378-403.

Touron P. (2004), « L'adoption des principes comptables généralement reconnus aux États-Unis par Saint-Gobain-Pont-à-Mousson : du conflit d'agence à la légitimité institutionnelle », Comptabilité - Contrôle - Audit, tome 10, numéro thématique, p. 161-191.

Watts R.L. et Zimmerman J.L. (1978), « Towards a Positive Theory of the Determination of Accounting Standards », Accounting Review, vol. 53, n 1, p. 112-134.

Watts R.L. et Zimmerman J.L. (1979), « The Demand for and Supply of Accounting Theories: The Market for Excuses », Accounting Review, vol. 54, n 2, p. 273-305.

Watts R.L. et Zimmerman J.L. (1986), Positive Accounting Theory, Prentice-Hall.

Watts R.L. et Zimmerman J.L. (1990), « Positive Accounting Theory: A Ten Year Perspective », Accounting Review, vol. 65, $\mathrm{n}^{\mathrm{0}}$ 1, p. 131-156.

Wegener M. et Labelle R. (2017), « Value Relevance of Environmental Provisions Pre- and PostIFRS », Accounting Perspectives, vol. 16, nº 3, p. 139-168.

Yin R.K. (2009), Case Study Research: Design and Methods, Sage Publications. 


\section{Annexes}

\section{Annexe 1}

Informations concernant les groupes interrogés (montants en millions d'euros, exercice 2010, les entretiens ayant eu lieu en 2011)

\begin{tabular}{|c|c|c|c|c|c|c|c|c|c|}
\hline Id. & Secteur d'activité & $\begin{array}{c}\text { Prov. } \\
\text { environ. }{ }^{1}\end{array}$ & Résultat & $\begin{array}{l}\text { Chiffre } \\
\text { d'affaires }\end{array}$ & $\begin{array}{l}\text { Total des } \\
\text { actifs }\end{array}$ & $\begin{array}{l}\text { Indice } \\
\text { boursier }\end{array}$ & $\begin{array}{l}\text { Groupe soumis aux } \\
\text { risques } \\
\text { environnementaux }\end{array}$ & $\begin{array}{c}\text { Personnes interrogées } \\
\text { (* entretien en totalité ou en partie collectif) }\end{array}$ & $\begin{array}{l}\text { Durée de } \\
\text { l'entretien }\end{array}$ \\
\hline \multirow{2}{*}{1.} & \multirow{2}{*}{ Pétrole } & \multirow{2}{*}{6786} & \multirow{2}{*}{10807} & \multirow{2}{*}{159269} & \multirow{2}{*}{143718} & \multirow{2}{*}{ CAC 40} & \multirow{2}{*}{ Oui } & Responsable du reporting environnement* & $20 \mathrm{~min}$ \\
\hline & & & & & & & & Responsable du reporting accident et sécurité* & $55 \mathrm{~min}$ \\
\hline 2. & Pharmaceutique & 781 & 5721 & 30384 & 85264 & CAC 40 & Oui & Directeur hygiène, sécurité, environnement & $1 \mathrm{~h} 5 \mathrm{~min}$ \\
\hline \multirow{3}{*}{3.} & \multirow{3}{*}{ Déchets et eau } & \multirow{3}{*}{757,1} & \multirow{3}{*}{871,6} & \multirow{3}{*}{34786,6} & \multirow{3}{*}{51511,3} & \multirow{3}{*}{ CAC 40} & \multirow{3}{*}{ Oui } & Directeur des risques* & $1 \mathrm{~h} 10 \mathrm{~min}$ \\
\hline & & & & & & & & $\begin{array}{l}\text { Directeur des indicateurs et de la performance } \\
\text { environnementale, sociale et de gouvernance* }\end{array}$ & $55 \mathrm{~min}$ \\
\hline & & & & & & & & Directeur financier de la filiale italienne & $5 \mathrm{~min}$ \\
\hline 4. & Déchets et eau & 560,9 & 720,1 & 13869,3 & 25930,4 & CAC 40 & Oui & Directeur du développement durable & $1 \mathrm{~h} 5 \mathrm{~min}$ \\
\hline \multirow{3}{*}{5.} & \multirow{3}{*}{ Télécommunications } & \multirow{3}{*}{507} & \multirow{3}{*}{4877} & \multirow{3}{*}{45503} & \multirow{3}{*}{94276} & \multirow{3}{*}{ CAC 40} & \multirow{3}{*}{ Oui } & Directeur des normes comptables* & \multirow{3}{*}{$35 \mathrm{~min}$} \\
\hline & & & & & & & & Collaborateur du directeur des normes comptables* & \\
\hline & & & & & & & & Directeur environnement* & \\
\hline 6. & Chimie & 271 & 262 & 5226 & 5131 & SBF 120 & Oui & Directeur de la réhabilitation environnementale & $1 \mathrm{~h} 10 \mathrm{~min}$ \\
\hline \multirow{3}{*}{7.} & \multirow{3}{*}{ Mine, métallurgie } & \multirow{3}{*}{123,9} & \multirow{3}{*}{245,3} & \multirow{3}{*}{4426,8} & \multirow{3}{*}{3346,7} & \multirow{3}{*}{ SBF 120} & \multirow{3}{*}{ Oui } & Directeur de la consolidation et du reporting* & $1 \mathrm{~h} 10 \mathrm{~min}$ \\
\hline & & & & & & & & Directeur financier* & $1 \mathrm{~h}$ \\
\hline & & & & & & & & $\begin{array}{l}\text { Directeur général de l'innovation, de la recherche } \\
\text { et de la technologie* }\end{array}$ & $55 \mathrm{~min}$ \\
\hline 8. & Automobile & 40 & 3490 & 38971 & 70107 & CAC 40 & Oui & Directeur de la comptabilité & $20 \mathrm{~min}$ \\
\hline \multirow{2}{*}{9.} & \multirow{2}{*}{ Automobile } & 2457 & 384 & 9632 & 7440 & SRF 120 & Oui & Directeur du développement durable & $1 \mathrm{~h} 30 \mathrm{~min}$ \\
\hline & & 24,51 & 384 & 9632 & 7440 & SBF 120 & Ou1 & Assistant du directeur du développement durable & $45 \mathrm{~min}$ \\
\hline 10. & Déchets & 12,6 & 26,9 & 402,12 & 751,84 & SBF 250 & Oui & Directeur du développement durable & $1 \mathrm{~h} 55 \mathrm{~min}$ \\
\hline
\end{tabular}

1. Provisions environnementales totales comprenant les provisions pour risques environnementaux et les provisions pour remise en état de site. 
Annexe 2

Cabinets d'audit interrogés

\begin{tabular}{|c|l|l|l|l|}
\hline Id. & \multicolumn{1}{|c|}{ Secteur d'activité } & \multicolumn{1}{|c|}{ Personnes interrogées } & \multicolumn{1}{c|}{$\begin{array}{c}\text { Durée de } \\
\text { l'entretien }\end{array}$} \\
\hline 11. & Audit (Big Four) & Commissaire aux comptes associé & $\begin{array}{l}\text { Associé certifiant les comptes de groupes cotés ayant des } \\
\text { provisions environnementales importantes }\end{array}$ & \multicolumn{1}{c}{1 h 15 min } \\
\hline 12. & Audit (Big Four) & $\begin{array}{l}\text { Commissaire aux comptes associé et } \\
\text { directeur du service développement durable }\end{array}$ & $\begin{array}{l}\text { Associé certifiant les comptes de groupes cotés ayant des } \\
\text { provisions environnementales importantes }\end{array}$ & $\begin{array}{l}\text { Commissaire aux comptes investi dans le développement de } \\
\text { la comptabilité environnementale en France }\end{array}$ \\
\hline \multirow{2}{*}{ 13. } & $\begin{array}{l}\text { Expertise comptable et } \\
\text { audit (province) }\end{array}$ & $\begin{array}{l}\text { Expert-comptable et commissaire aux } \\
\text { comptes }\end{array}$ & $\begin{array}{l}\text { Spécialisée dans les audits d'informations } \\
\text { environnementales }\end{array}$ \\
\cline { 3 - 5 } & Collaboratrice & \multicolumn{2}{|c|}{35 min } \\
\hline
\end{tabular}




\section{Annexe 3a}

Guide d'entretien à destination des directeurs comptables

1. Éléments de définition des risques environnementaux

- Que recouvre la notion de « risque environnemental » dans votre entreprise ? Quels sont vos risques environnementaux?

- Comment sont détectés et estimés ces risques ? Pouvez-vous me décrire votre démarche de management des risques environnementaux ?

- Comment est abordé l'horizon temporel des risques environnementaux ? Quel est-il ?

2. Rythme/cycle du processus de reporting financier

- Avant d'aller plus dans le détail de la démarche de reporting des risques environnementaux, pouvez-vous me parler de la démarche générale de reporting financier? Quelles étapes, quelles personnes impliquées, quel rythme?

- Comment se place le processus relatif au reporting des risques environnementaux dans ce cadre-là ?

- Quelles sont les étapes de cette décision?

- Quelles procédures sont suivies?

- Qui est responsable de l'information communiquée ?

- Abordez-vous la décision de provisionner ces risques de la même manière pour chaque type de risque environnemental ?

- En général, avez-vous une idée de quelles personnes ce genre d'information peut intéresser ? Est-ce que vous avez des demandes spécifiques relatives à ce genre d'informations ?

- Quels sont les enjeux relatifs à ce type de risque, selon vous ?

- Quels sont les utilisateurs les plus attentifs à ce type d'information ?

- Les analystes financiers y font-ils attention (dans le cadre des réunions que vous pouvez avoir avec eux)?

3. Acteurs et responsabilités

- Qui participe à la décision de provisionner ces risques ou d'insérer une information sur ces risques?

- Qui sont les réels décisionnaires ?

- Quels sont les personnes, internes ou externes à l'entreprise, qui exercent une certaine influence sur cette décision?

- Quel est votre rôle direct ou indirect dans ce processus décisionnel ?

- Quel rôle jouent les autres acteurs?

- Quelles tactiques politiques, quels jeux d'acteurs, quels rapports de forces percevez-vous entre les acteurs que vous venez de citer sur ces questions de provisions pour risques environnementaux?

4. Déterminants de la décision de provisionnement

- En fonction de quels critères cette décision de provisionner est-elle prise ?

- Quels facteurs, déterminants, sont susceptibles d'influencer l'issue de la décision?

- Quel a été l'impact de la loi NRE sur la comptabilisation de ce genre de risques ?

- Quel a été l'impact du passage aux IFRS sur la comptabilisation de ce genre de risques ?

- Comparez-vous vos pratiques en la matière avec celles de groupes similaires au vôtre ? Quelles influences ces comparaisons ont sur vos décisions? 


\section{Annexe 3b}

Guide d'entretien à destination des commissaires aux comptes

1. Éléments de définition des risques environnementaux

- Que recouvre la notion de « risque environnemental » lorsque vous auditez ? Quels sont les risques environnementaux qui reviennent le plus souvent?

2. Démarche d'audit des risques environnementaux et des provisions associées

- Comment appréciez-vous les provisions pour risques environnementaux des entreprises que vous auditez?

- Comment intervenez-vous dans la décision de provisionner certains de ces risques ?

- Quelle est votre démarche d'audit pour ces provisions?

- Qui sont vos interlocuteurs?

- Quels sont les enjeux relatifs à ce type de risques et de provisions ?

- Quels problèmes rencontrez-vous concernant ces provisions?

3. Décision de provisionner au sein des groupes

- Que savez-vous des étapes de la décision de provisionnement des risques dans les groupes que vous auditez?

- Quelles procédures sont suivies?

- Qui est responsable de l'information communiquée?

- Y a-t-il des différences de procédures entre les différents types de risque environnemental ?

- En général, avez-vous une idée des personnes que ce genre d'informations peut intéresser ? Est-ce que les entreprises ont des demandes spécifiques relatives à ce genre d'informations?

- Quels sont les utilisateurs les plus attentifs à ce type d'informations ?

- Les analystes financiers y font-ils attention?

4. Acteurs et responsabilités

- Qui participe à la décision de provisionner ces risques ou d'insérer une information sur ces risques?

- Qui sont à votre avis les réels décisionnaires ?

- Quels sont les acteurs, internes ou externes à l'entreprise, qui exercent une certaine influence sur cette décision?

- Quel est votre rôle direct ou indirect dans ce processus décisionnel ?

- Quel rôle jouent les autres acteurs ?

- Quels rapports de forces percevez-vous entre les acteurs que vous venez de citer sur ces questions de provisions pour risques environnementaux ?

5. Déterminants de la décision de provisionnement

- En fonction de quels critères cette décision de provisionner est-elle prise ?

- Quels facteurs, déterminants, sont susceptibles d'influencer l'issue de la décision?

- Quel a été l'impact de la loi NRE sur la comptabilisation de ce genre de risques ?

- Quel a été l'impact du passage aux IFRS sur la comptabilisation de ce genre de risques ?

- Est-ce que vous faites un suivi dans le temps des provisions environnementales enregistrées au regard des réalisations effectives? 
Annexe 4

Nœuds de codage des entretiens sur NVivo

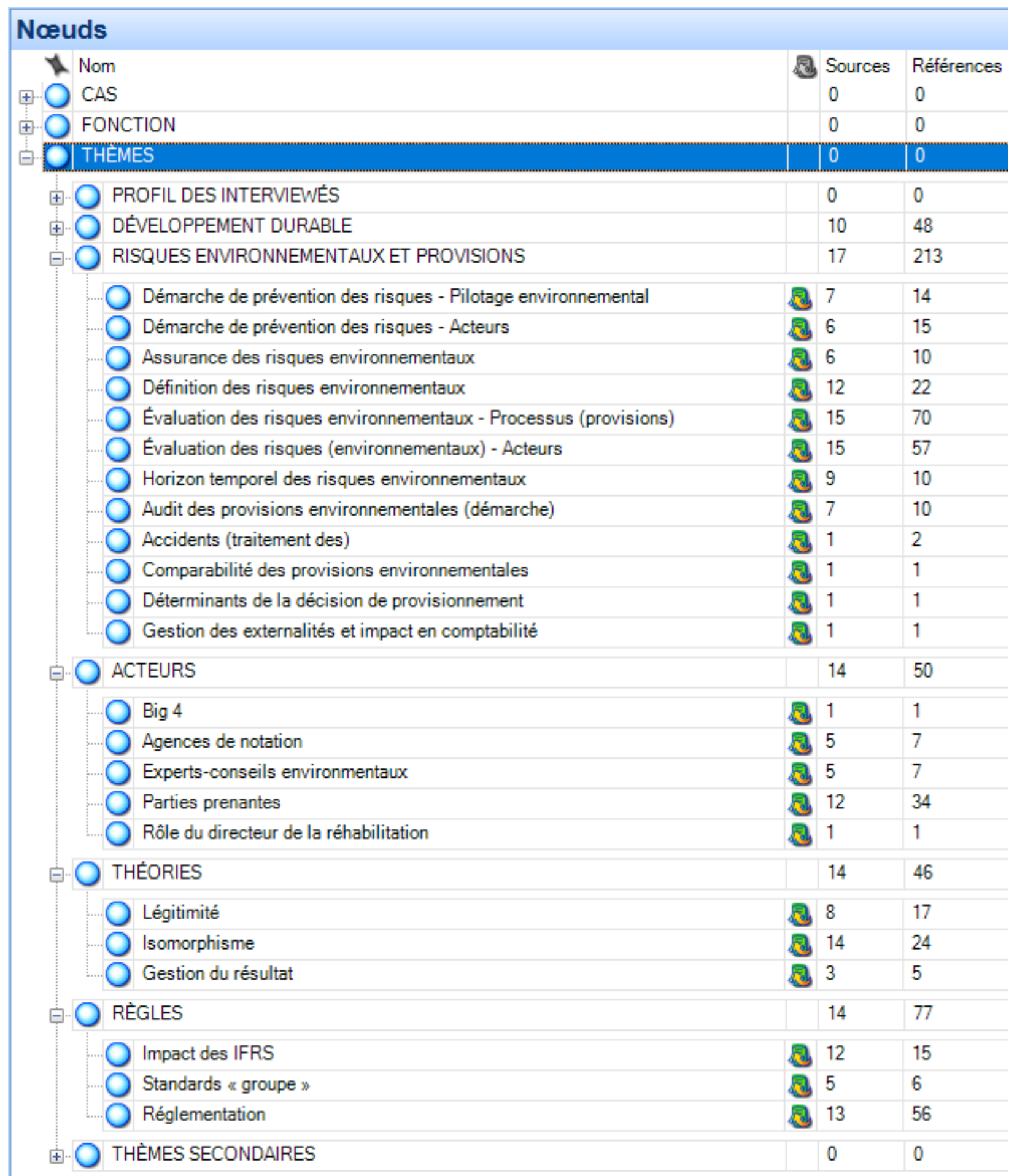

Des mémos récapitulatifs ont été attachés à chaque nœud afin de faire la synthèse des discours retranscrits. Ces mémos ont servi de base à l'écriture et l'analyse des résultats. 